\title{
Solving the complete-electrode direct model of ERT using the boundary element method and the method of fundamental solutions
}

T. E. Dyhoum ${ }^{1,2}$, D. Lesnic ${ }^{1}$, and R. G. Aykroyd ${ }^{2}$

${ }^{1}$ Department of Applied Mathematics, ${ }^{2}$ Department of Statistics, University of Leeds, Leeds LS2 9JT, UK

E-mails: mmted@leeds.ac.uk, D.Lesnic@leeds.ac.uk, R.G.Aykroyd@leeds.ac.uk

\begin{abstract}
This paper discusses solving the forward problem for electrical resistance tomography (ERT). The mathematical model is governed by Laplace's equation with the most general boundary conditions forming the so-called completeelectrode model (CEM). We examine this problem in simply-connected and multiply - connected domains (rigid inclusion, cavity and composite bi-material). This direct problem is solved numerically using the boundary element method (BEM) and the method of fundamental solutions (MFS). The resulting BEM and MFS solutions are compared in terms of accuracy, convergence and stability. Anticipating the findings, we report that the BEM provides a convergent and stable solution, whilst the MFS places some restrictions on the number and location of the source points.
\end{abstract}

Keywords: Electrical impedance/resistance tomography; Complete-electrode model; Boundary element method; Method of fundamental solutions.

\section{Introduction}

Electrical impedance tomography (EIT) is a non-intrusive, low-cost and portable technique of imaging the interior of a specimen based on the knowledge of the injected currents and the resulted voltages which are measured on the electrodes, as explained in $[25,26]$. It has widespread applications in medicine, geophysics and industry. When using this technique in electrostatics, for example, one seeks to create images of the electrical conductivity distribution in the body from static electric measurements on the boundary of the body, [18]. The EIT direct (forward) problem provides the current flux on the boundary, which in turn, leads to calculation of voltages, via an estimated conductivity distribution. In contrast, the inverse EIT problem aims to reconstruct the inner conductivity distribution from the knowledge of the voltages for a wide pattern of injecting currents. In this iterative optimization process, the nonlinear least-squares objective function has to be evaluated many times using the forward solver. Consequently, there is a need to obtain the solution of the direct problem accurately and fast if it is to be useful for real-time monitoring, [11,12,22, 23].

If all the quantities involved are real then, this version of the more general complex EIT is also known as electrical resistance tomography (ERT). We mention that some comparison has been previously performed in [10] between the finite 
volume method (FVM) and the finite element method (FEM), for the gap model of EIT. Also, very recently on improved boundary distributed source method has been compared in [8] with the more standard BEM and FEM numerical forward solvers for ERT.

We begin with the mathematical formulation (Section 2) which describes the complete-electrode model (CEM) for ERT. Since in the direct problem of the CEM the constant voltage on each electrode is unknown, we can eliminate it by integrating the associated Robin boundary condition, as described in [9]. The resulting mathematical model is then solved using two numerical methods. These are the boundary element method (BEM) (Section 3) and, for the first time, the meshless method of fundamental solutions (MFS) (Section 4). In the same spirit as [8], we compare thoroughly the numerical results obtained by these two methods for both simply-connected (Section 5) and multiply-connected domains containing a rigid inclusion or a cavity (Section 6). An extension to composite bi-materials is also performed (Section 7). Finally, the conclusions of this study (Section 8) are given paving the way for future work on solving the inverse problem of ERT/EIT.

\section{Mathematical Formulation}

In this section, we consider solving Laplace's equation in a two-dimensional bounded domain $\Omega$, namely,

$$
\nabla^{2} u=0, \quad \text { in } \Omega,
$$

subject to certain boundary conditions which make the problem the so-called 'complete-electrode model' (CEM), [24]. In this model, on the boundary $\partial \Omega$ there are attached $L$ electrodes, $\varepsilon_{p}$, for $p=\overline{1, L}$, see Figure 1 . On these electrodes we have the Robin boundary condition, [9],

$$
u+z_{p} \frac{\partial u}{\partial n}-\frac{1}{\ell_{p}} \int_{\varepsilon_{p}} u d s=\frac{z_{p} I_{p}}{\ell_{p}}, \quad \text { on } \varepsilon_{p}, \quad p=\overline{1, L},
$$

where $\underline{n}$ is the outward unit normal to the boundary $\partial \Omega, \ell_{p}$ is the length of the electrode $\varepsilon_{p}$,

$$
I_{p}=\int_{\varepsilon_{p}} \frac{\partial u}{\partial n} d s
$$

is the injected constant current applied on the electrode $\varepsilon_{p}$ and satisfying $\sum_{p=1}^{L} I_{p}=$ 0 , and $z_{p}>0$ is the constant contact impedance. In equations (1)-(3) we have assumed that the medium $\Omega$ has unit constant conductivity, but later on we shall also consider a piecewise constant version. The derivation of the boundary condition (2) is as follows. The constant voltages $U_{p}$ on the electrodes $\varepsilon_{p}$, that are to 
be determined in the direct problem, are calculated in the inverse problem from the Robin boundary condition

$$
u+z_{p} \frac{\partial u}{\partial n}=U_{p}, \quad \text { on } \varepsilon_{p}, p=\overline{1, L} .
$$

Then, by integrating (4) over $\varepsilon_{p}$, and using (3) we can eliminate the unknown $U_{p}$ to obtain (2).

The electric current is assumed to vanish on the gaps $g_{p}$ for $p=\overline{1, L}$, between the electrodes on the boundary part, so that

$$
\frac{\partial u}{\partial n}=0, \quad \text { on } \partial \Omega \backslash \cup_{p=1}^{L} \varepsilon_{p}=: \cup_{p=1}^{L} g_{p} .
$$

In order to obtain a unique solution we also need that, [1],

$$
\int_{\partial \Omega} u d s=0 .
$$

Equations (1), (2), (5) and (6) represent the direct problem of ERT if the domain $\Omega$ is simply-connected. If $\Omega$ is multiply-connected, e.g. it contains holes, then an additional boundary condition of the form

$$
u=0, \quad \text { or } \quad \frac{\partial u}{\partial n}=0, \quad \text { or } \quad z \frac{\partial u}{\partial n}+u=0
$$

should be applied on the inner boundary portions of $\partial \Omega$, where $z \geq 0$ is the contact impedance.

The CEM given by equations (1), (3)-(6) is uniquely solvable, [24], and has been validated in $[8]$ as being in most agreement with experiments compared with the simpler continuous, gap and shunt models of ERT/EIT.

Without loss of generality, we can assume that $\Omega$ is the unit disk $\{(x, y) \in$ $\left.\mathbb{R}^{2} \mid x^{2}+y^{2}<1\right\}$, otherwise we can always map conformally the simply-connected domain $\Omega$ onto the unit disk.

A closed form solution of the direct problem of ERT is available only in very restricted cases, e.g. for $L=2$ electrodes, and no-impedances $z_{1}=z_{2}=0$, [20], and therefore numerical methods are generally necessary. In the next sections we describe and compare two such numerical methods. 
Figure 1: The electrodes attached to the boundary in two-dimensional CEM for $L=2$ and 4 .
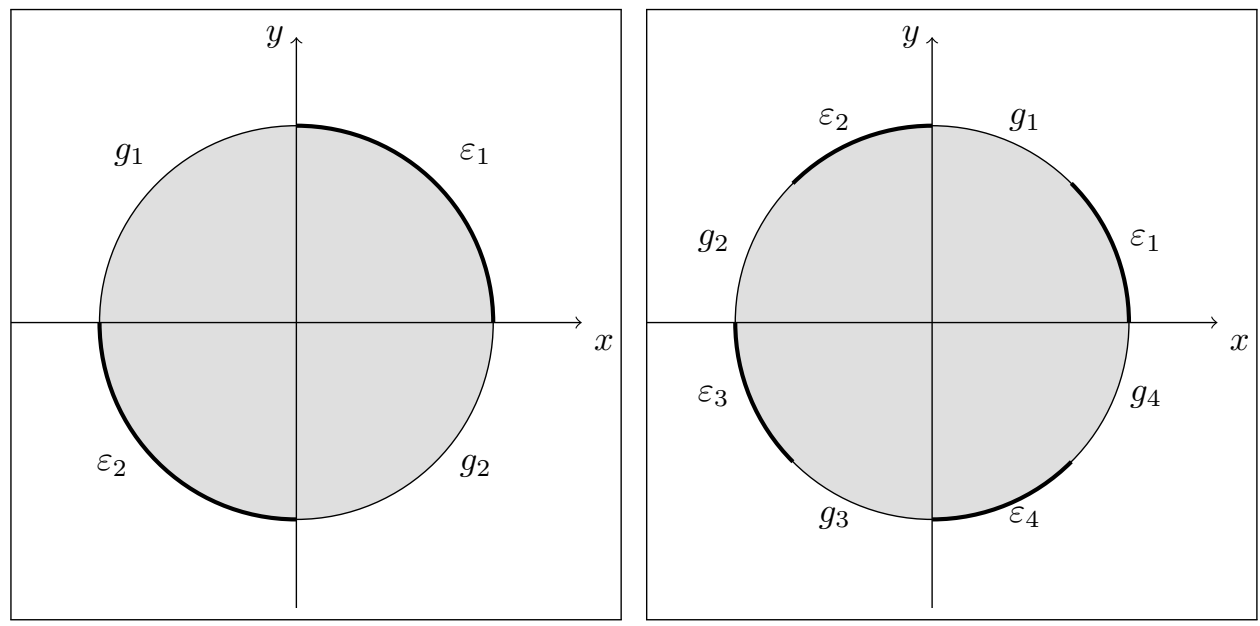

\section{The Boundary Element Method}

The boundary element method (BEM) has many advantages compared to other domain discretisation methods because it discretises only the boundary to obtain the unspecified boundary data and the solution in the whole domain, [2,13]. This reduction makes the number of unknowns, which need to be determined, smaller. In this section, we will use the BEM to solve the forward problem (1), (2), (5) and (6) in the unit disk $\Omega=\left\{(x, y) \in \mathbb{R}^{2} \mid x^{2}+y^{2}<1\right\}$. The BEM reduces the problem to one of solving a linear system of equations

$$
A \underline{u^{\prime}}+B \underline{u}=\underline{0}
$$

where $\underline{u}:=\left(u\left(\underline{\tilde{p}}_{j}\right)\right)_{j=\overline{1, M}}, \underline{u}^{\prime}:=\left(\frac{\partial u}{\partial n}\left(\underline{\tilde{p}}_{j}\right)\right)_{j=\overline{1, M}}, A$ and $B$ are matrices which depend solely on the geometry of $\partial \Omega$, and $M$ is the number of boundary elements. The boundary element endpoint is $\underline{p_{j}}=\left(x_{j}, y_{j}\right)=\left(\cos \left(\frac{2 \pi j}{M}\right), \sin \left(\frac{2 \pi j}{M}\right)\right)$, for $j=\overline{1, M}$, with the convention that $\underline{p}_{0}=\underline{p}_{M}$ and $\tilde{p}_{j}$ is the boundary element node. For a constant BEM approximation $\underline{\tilde{\tilde{p}}}_{j}$ is the midpoint of the segment $\Gamma_{j}=\bar{p}_{j-1}, \underline{p}_{j}$. The derivation of this approximation can be briefly summarised in the following four steps:

1. Find the fundamental solution $G\left(p, p^{\prime}\right)$ of Laplace's equation satisfying

$$
\nabla^{2} G\left(\underline{p}, \underline{p^{\prime}}\right)=-\delta\left(\underline{p}-\underline{p^{\prime}}\right),
$$


where $\delta$ is the Dirac delta function. The fundamental solution which we seek is based on the distance between $\underline{p}$ and $\underline{p}^{\prime}$. As a result, in two-dimensions

$$
G\left(\underline{p}, \underline{p}^{\prime}\right)=-\frac{1}{2 \pi} \ln \left|\underline{p}-\underline{p^{\prime}}\right|=-\frac{1}{2 \pi} \ln \sqrt{\left(x-x^{\prime}\right)^{2}+\left(y-y^{\prime}\right)^{2}},
$$

where $\underline{p}=(x, y)$ and $\underline{p}^{\prime}=\left(x^{\prime}, y^{\prime}\right)$.

2. Transform Laplace's equation into the integral equation

$$
\eta(\underline{p}) u(\underline{p})=\int_{\partial \Omega}\left[G\left(\underline{p}, \underline{p^{\prime}}\right) \frac{\partial u}{\partial n}-u\left(\underline{p^{\prime}}\right) \frac{\partial G}{\partial n}\left(\underline{p}, \underline{p^{\prime}}\right)\right] d S,
$$

where

$$
\eta(\underline{p})= \begin{cases}0.5 & \text { if } \underline{p} \in \partial \Omega \text { (smooth) } \\ 1 & \text { if } \underline{p} \in \Omega \\ 0 & \text { if } \underline{p} \notin \bar{\Omega} .\end{cases}
$$

This is obtained using the fundamental solution (9) and Green's identity.

3. Discretise the boundary into small straight line segments $\Gamma_{j}$ for $j=\overline{1, M}$ and assume that the boundary potential $u$ and its normal derivative $\frac{\partial u}{\partial n}$ are approximated by constant functions over each small boundary element $\Gamma_{j}$. Via these approximations, the integral equation (10) is expressed as

$$
\eta(\underline{p}) u(\underline{p})=\sum_{j=1}^{M} u_{j}^{\prime} A_{j}(\underline{p})-\sum_{j=1}^{M} u_{j} B_{j}(\underline{p}),
$$

where

$$
\begin{array}{rlr}
A_{j}(\underline{p}) & =\int_{\Gamma_{j}} G\left(\underline{p}, \underline{p^{\prime}}\right) d \Gamma_{j}\left(\underline{p^{\prime}}\right) \\
& =-\frac{1}{2 \pi} \begin{cases}h(\ln (h / 2)-1) & \text { if } a b=0 \\
a \cos (\beta)(\ln (a)-\ln (b))-h(1-\ln (b))+a \psi \sin (\beta) & \text { if } a b \neq 0\end{cases} \\
B_{j}(\underline{p}) & =\int_{\Gamma_{j}} \frac{\partial G}{\partial n}\left(\underline{p}, \underline{p^{\prime}}\right) d \Gamma_{j}\left(\underline{p^{\prime}}\right) & \\
& =\frac{1}{2 \pi} \begin{cases}0 & \text { if } a b=0 \text { or } \underline{p} \in\left\{\underline{p}_{j-1}, \underline{p}_{j}\right\} \\
\psi \operatorname{sign}\left(\alpha_{j-1}(\underline{p})-\alpha_{j}(\underline{p})\right) & \text { if } y \in\left[y_{j-1}, y_{j}\right] \\
\psi \operatorname{sign}\left(\alpha_{j}(\underline{p})-\alpha_{j-1}(\underline{p})\right) & \text { otherwise }\end{cases}
\end{array}
$$

where sign is the signum function, $a=\left|\underline{p}-\underline{p}_{j-1}\right|, b=\left|\underline{p}-\underline{p}_{j}\right|, h=\left|\underline{p}_{j}-\underline{p}_{j-1}\right|$, $\alpha_{j-1}(\underline{p})$ and $\alpha_{j}(\underline{p})$ are the angles between the $x$-axis and segments $\underline{\underline{p}, \underline{p}} \underline{p}_{j-1}$ and $\underline{\underline{p}, \underline{p}}$, repectively, and the angles $\psi$ and $\beta$ are given by

$$
\psi=\arccos \left(\frac{a^{2}+b^{2}-h^{2}}{2 a b}\right), \beta=\arccos \left(\frac{a^{2}+h^{2}-b^{2}}{2 a h}\right) .
$$


4. Apply equation (11) at the midpoint nodes $\tilde{p}_{i}$ for $i=\overline{1, M}$. This gives the system of linear algebric equations (8) with the unknowns $\underline{u}$ and $\underline{u}^{\prime}$. The system can be rewritten as

$$
\sum_{j=1}^{M}\left(A_{i j} u_{i}^{\prime}+B_{i j} u_{i}\right)=\underline{0}, \quad i=\overline{1, M},
$$

where $A$ and $B$ are matrices defined by

$$
A_{i j}=A_{j}\left(\underline{\tilde{p}_{i}}\right), \quad B_{i j}=-B_{j}\left(\underline{\tilde{p}_{i}}\right)-\frac{1}{2} \delta_{i j},
$$

where $\delta_{i j}$ is the Kronecker delta function.

In compact form (12) represents the system of equations (8). Specific boundary conditions should be imposed to make the resulting system of equations (12) solvable. The CEM boundary conditions (2), (5) and (6) will be considered next.

First, we collocate the boundary condition (2) for the electrodes $\varepsilon_{p}, p=\overline{1, L}$, at the nodes $\tilde{p}_{i}$, resulting in

$$
\begin{aligned}
u_{i}+z_{p} u_{i}^{\prime}-\frac{2 \pi}{M \ell_{p}} \sum_{k=(K M / L)+1}^{(2 K+1) M /(2 L)} u_{k} & =\frac{z_{p} I_{p}}{\ell_{p}}, \\
i & =\overline{(M+1+K M / L),(M+(2 K+1) M /(2 L))},
\end{aligned}
$$

where $K=\overline{0,(L-1)}$. This yields $M / 2$ equations.

Secondly, by collocating the zero flux boundary condition (5) for the gaps $g_{p}$, $p=\overline{1, L}$, between electrodes at the nodes $\tilde{p}_{i}$, we obtain

$$
u_{i}^{\prime}=0, \quad i=\overline{(M+1+(2 K-1) M /(2 L)),(M+K M / L)},
$$

where $K=\overline{1, L}$. This yields another $M / 2$ equations.

Finally, the condition (6) yields one more equation, namely,

$$
\sum_{k=1}^{M} u_{k}=0
$$

To find the solution of the CEM problem (1), (2), (5) and (6) using the BEM, the 
equations (12)-(15) have been reformulated in the following generic matrix form as a $(2 M+1) \times(2 M)$ linear system of algebraic equations:

$$
D \underline{X}=\underline{b}
$$

where

$$
\underline{X}=\left(\frac{\underline{u}}{u^{\prime}}\right) .
$$

Of course, from equations (13) and (14), in principle we could eliminate the current flux $\underline{u}^{\prime}$ such that (16) can be reduced to a smaller $(M+1) \times M$ linear system of algebraic equations. Since the system of equations (16) is over-determined (the number of equations is greater than the number of unknowns), we can use the least-squares method to solve it. This yields

$$
\underline{X}=\left(D^{T} D\right)^{-1} D^{T} \underline{b} .
$$

Once the boundary values have been obtained accurately, equation (11) can be applied at $\underline{p} \in \Omega$ to provide explicitly the interior solution for $u(\underline{p})$.

\section{The Method of Fundamental Solutions}

One of the reasons why the method of fundamental solutions (MFS) is becoming increasingly popular in various applications is that it is conceptually simple and easy to describe and implement. The MFS is regarded as a meshless BEM and it has been used to find the solution of inverse geometric problems governed by the Laplace's equation in $[14,15]$.

The MFS seeks a solution of Laplace's equation (1) as a linear combination of fundamental solutions of the form:

$$
u(\underline{p})=\sum_{j=1}^{N} c_{j} G\left(\underline{\xi}_{j}, \underline{p}\right), \quad \underline{p} \in \bar{\Omega}=\Omega \cup \partial \Omega,
$$

where $\underline{\xi}_{j}$ are called sources ('singulaties') and are located outside $\bar{\Omega}$, and $\left(c_{j}\right)_{j=\overline{1, N}}$ are unknown coefficients to be determined by imposing the boundary conditions (2), (5) and (6). The approximation (18) is justified by the denseness of the set of these functions, as $N \rightarrow \infty$, into the set of harmonic functions, [6]. Note that in $\mathbb{R}^{2}$ there is an additional constant which has to be included in the expression (18) in order for the set to be complete, but this constant can usually be taken to be zero without much loss of generality.

Since $\Omega$ is the unit disk, we take the source points

$$
\underline{\xi}_{j}=\left(\xi_{j}^{1}, \xi_{j}^{2}\right)=\left(R \cos \left(\frac{2 \pi j}{N}\right), R \sin \left(\frac{2 \pi j}{N}\right)\right), \quad j=\overline{1, N},
$$


where $1<R<\infty$, and the boundary collocation points

$$
\underline{x}_{i}=\left(\cos \left(\frac{2 \pi i}{M}\right), \sin \left(\frac{2 \pi i}{M}\right)\right), \quad i=\overline{1, M} .
$$

From (9) we have

$$
\frac{\partial G}{\partial n}\left(\underline{\xi}_{j}, \underline{p}\right)=\frac{1-\left(\xi_{j}^{1} x+\xi_{j}^{2} y\right)}{2 \pi\left|\underline{\xi}_{j}-\underline{p}\right|^{2}}, \quad \underline{p}=(x, y) \in \partial \Omega
$$

where $\underline{\xi}_{j}=\left(\xi_{j}^{1}, \xi_{j}^{2}\right)$. In order to obtain the coefficient vector $\underline{c}=\left(c_{j}\right)_{j=\overline{1, N}}$, we substitute equations (9) and (19) into the boundary conditions (2), (5) and (6).

Firstly, we apply the boundary condition (2) for the electrodes $\varepsilon_{p}, p=\overline{1, L}$, at the collocation points $\underline{x}_{i}$ on $\varepsilon_{p}$ resulting in

$$
\begin{array}{r}
\sum_{j=1}^{N}\left[G\left(\underline{\xi}_{j}, \underline{x}_{i}\right)-\frac{2 \pi}{M \ell_{p}} \sum_{k=(K M / L)+1}^{(2 K+1) M /(2 L)} G\left(\underline{\xi}_{j}, \underline{x}_{k}\right)+z_{p} \frac{\partial G}{\partial r}\left(\underline{\xi}_{j}, \underline{x}_{i}\right)\right] c_{j}=\frac{z_{p} I_{p}}{\ell_{p}} \\
i=\overline{(K M / L)+1,(2 K+1) M /(2 L)}
\end{array}
$$

where $K=\overline{0,(L-1)}$. This yields $M / 2$ equations.

Secondly, by applying the zero flux boundary condition (5) on the gaps $g_{p}, p=$ $\overline{1, L}$, between electrodes, at the collocation points $\underline{x}_{i}$ on $g_{p}$, we obtain

$$
\sum_{j=1}^{N} c_{j} \frac{\partial G}{\partial r}\left(\underline{\xi}_{j}, \underline{x}_{i}\right)=0, \quad i=\overline{(1+(2 K-1) M /(2 L)),(K M / L)},
$$

where $K=\overline{1, L}$. This yields another $M / 2$ equations.

Finally, imposing the condition (6) yields one more equation

$$
\sum_{i=1}^{M} \sum_{j=1}^{N} c_{j} G\left(\underline{\xi}_{j}, \underline{x}_{i}\right)=0
$$

Again, to find the solution of the CEM problem (1), (2), (5) and (6) using the MFS, the equations (20)-(22) have been reformulated in the following generic matrix form as an $(M+1) \times N$ linear system of algebraic equations

$$
F \underline{c}=\underline{b} .
$$


The least-squares method is used to solve the system of equations (23) if $M+1 \geq$ $N$. This yields

$$
\underline{c}=\left(F^{T} F\right)^{-1} F^{T} \underline{b} .
$$

Once the coefficient vector $\underline{c}$ has been obtained accuratly, equations (18) and (19) provide explicitly the solution for the potential $u$ in $\bar{\Omega}$, and the current flux $\partial u / \partial n$ on $\partial \Omega$.

\section{Numerical Results and Discussion}

In this section, we will discuss and compare the numerical solutions of the direct ERT problem given by equations (1), (2), (5) and (6) obtained using the BEM and the MFS.

Example 1. For simplicity, choose $L=2$ (only two electrodes are attached to the boundary) and solve the problem (1), (2), (5) and (6) with the following input data: $z_{1}=z_{2}=I_{1}=1$, and $I_{2}=-1$.

BEM Solution: The matrix $D$ in equation (16) is given by



On the other hand, using equations (13)-(15) we obtain:

$$
\begin{gathered}
D_{i, l}= \begin{cases}-\frac{h}{\ell_{1}} & \text { if }(i-M) \neq l, l=\overline{1, M / 4}, \\
\left(1-\frac{h}{\ell_{1}}\right) & \text { if }(i-M)=l, l=\overline{1, M / 4}, \quad i=\overline{(M+1),(M+M / 4)} \\
0 & \text { if } l=\overline{(M / 4+1), M}, \\
z_{1} \delta_{i, l} & \text { if } l=\overline{(M+1), 2 M},\end{cases} \\
D_{i, l}= \begin{cases}-\frac{h}{\ell_{2}} & \text { if }(i-M) \neq l, l=\overline{(M / 2+1), 3 M / 4}, \\
\left(1-\frac{h}{\ell_{2}}\right) & \text { if }(i-M)=l, l=\overline{(M / 2+1), 3 M / 4}, \\
z_{2} \delta_{i, l} & \text { if } l=\overline{1, M / 2} \cup \overline{(3 M / 4+1), M}, \\
\text { if } l=\overline{(M+1), 2 M},\end{cases} \\
i=\overline{(M+M / 4+1),(M+M / 2) \cup \overline{(M+3 M / 4+1), 2 M} .}
\end{gathered}
$$


The last row in the matrix $D$ is given by

$$
D_{(2 M+1), l}= \begin{cases}1 & \text { if } l=\overline{1, M} \\ 0 & \text { if } l=\overline{M+1,2 M} .\end{cases}
$$

Finally, the vector $\underline{b}$ is given by

$$
\underline{b}=\left(\begin{array}{llllll}
\underline{0} & \frac{z_{1} I_{1}}{\ell_{1}} & \underline{0} & \frac{z_{2} I_{2}}{\ell_{2}} & \underline{0} & 0
\end{array}\right)^{T} .
$$

Table 1 illustrates the numerical solutions of the direct problem (1), (2), (5) and (6) obtained using the BEM with various numbers of boundary elements $M$. We only show the solution in the upper semidisk because the solution is symmetric on the lower semidisk, namely $u(x, y)=u(-x,-y)$ for $x \in(-1,1), y \in(0,1)$. Also, in Table 1 (as well as Tables 2 and 4 later on) we only show, for the simplicity of illustration, the results at $r \in\{1,2,3,9\} / 10$. We mention that the numerical results for the other values of $r \in\{4, \ldots, 8\} / 10$ have been found to possess similar features and therefore are not included. From Table 1 it can be seen that using the BEM to solve the CEM yields a convergent interior solution, as the number of boundary elements $M$ increases. 
Table 1: The numerical solution of Example 1 at selected interior points $(r, \theta)$ obtained using the BEM for various numbers of boundary elements $M \in\{8,16,32,64,128,256\}$.

\begin{tabular}{|c||c|c|c|c|c|c|}
\hline$r$ & $2 \pi / 10$ & $4 \pi / 10$ & $6 \pi / 10$ & $8 \pi / 10$ & $10 \pi / 10$ & $M$ \\
\hline \multirow{5}{*}{$1 / 10$} & 0.0540 & 0.0487 & 0.0247 & -0.0085 & -0.0386 & 8 \\
& 0.0556 & 0.0502 & 0.0255 & -0.0088 & -0.0398 & 16 \\
& 0.0561 & 0.0508 & 0.0257 & -0.0088 & -0.0401 & 32 \\
& 0.0562 & 0.0506 & 0.0257 & -0.0089 & -0.0401 & 64 \\
& 0.0562 & 0.0506 & 0.0257 & -0.0089 & -0.0402 & 128 \\
& 0.0562 & 0.0507 & 0.0258 & -0.0089 & -0.0402 & 256 \\
\hline \multirow{5}{*}{$2 / 10$} & 0.1083 & 0.974 & 0.0491 & -0.0169 & -0.0769 & 8 \\
& 0.1116 & 0.1004 & 0.0517 & -0.0174 & -0.0793 & 16 \\
& 0.1124 & 0.1011 & 0.0511 & -0.0175 & -0.0799 & 32 \\
& 0.1126 & 0.1013 & 0.0511 & -0.0176 & -0.0800 & 64 \\
& 0.1126 & 0.1014 & 0.0512 & -0.0176 & -0.0801 & 128 \\
& 0.1127 & 0.1014 & 0.0512 & -0.0176 & -0.0801 & 256 \\
\hline \multirow{5}{*}{$3 / 10$} & 0.1632 & 0.1463 & 0.0727 & -0.0248 & -0.1147 & 8 \\
& 0.1681 & 0.1508 & 0.0751 & -0.0257 & -0.1184 & 16 \\
& 0.1693 & 0.1519 & 0.0757 & -0.0259 & -0.1193 & 32 \\
& 0.1696 & 0.1521 & 0.0759 & -0.0260 & -0.1195 & 64 \\
& 0.1697 & 0.1522 & 0.0759 & -0.0260 & -0.1196 & 128 \\
& 0.1697 & 0.1522 & 0.0759 & -0.0260 & -0.1196 & 256 \\
\hline$\ldots$ & $\ldots$ & $\ldots$ & $\ldots$ & $\ldots$ & $\ldots$ & $\ldots$ \\
\hline \multirow{3}{*}{$9 / 10$} & 0.5051 & 0.4723 & 0.1592 & -0.0734 & -0.3175 & 8 \\
& 0.5264 & 0.4774 & 0.1793 & -0.0565 & -0.3440 & 16 \\
& 0.5264 & 0.4774 & 0.1793 & -0.0565 & -0.3440 & 32 \\
& 0.5263 & 0.4772 & 0.1792 & -0.0564 & -0.3436 & 64 \\
& 0.5264 & 0.4774 & 0.1793 & -0.0565 & -0.3439 & 128 \\
& 0.5264 & 0.4774 & 0.1793 & -0.0565 & -0.3440 & 256 \\
\hline
\end{tabular}

Figures 2 and 3 show the BEM boundary solution for $u$ and its normal derivative $\partial u / \partial n$, respectively. From these figures it can be seen that the BEM solutions for both $u$ and $\partial u / \partial n$ have rapid convergence on the boundary. So, we can rely on these results and consider them as the 'exact solution' of the well-posed direct problem of the CEM of EIT. 




Figure 2: The boundary solution $u(1, \theta)$, as a function of $\theta /(2 \pi)$, obtained using the BEM with $M \in\{64,128,256\}$, for Example 1 .



Figure 3: The normal derivative $\frac{\partial u}{\partial n}(1, \theta)$, as a function of $\theta /(2 \pi)$, obtained using the BEM with $M \in\{64,128,256\}$, for Example 1 .

Figure 4 shows the resulting voltage $U_{p}, p=1,2$, obtained from (4). In this figure the top part illustrates that the voltage is indeed constant and equal to $U_{1} \approx 1.1738$, whilst the bottom one indicates that $U_{2} \approx-1.1738$. We mention that these voltages are the quantities which are measured on the first and second electrodes in the inverse ERT problem. 


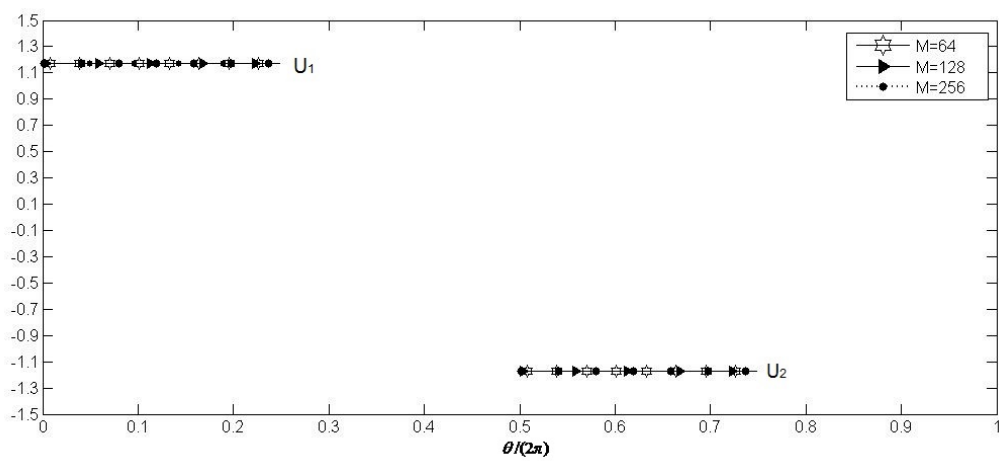

Figure 4: The voltages $U_{p}, p=1,2$, as functions of $\theta /(2 \pi)$, obtained using the BEM with $M \in\{64,128,256\}$, for Example 1 .

MFS solution: We now solve the problem (1), (2), (5) and (6) for Example 1 using the MFS instead of the BEM.

To begin with, the first $M / 4$ rows of the matrix $F$ in equation (23) coresponding to the first electrode $\varepsilon_{1}$ are

$F_{i, j}=G_{i, j}-\frac{2 \pi}{M \ell_{1}}\left(G_{i, j}+G_{i+1, j}+\ldots+G_{M / 4, j}\right)+z_{1} G_{i, j}^{\prime}, \quad i=\overline{1, M / 4}, \quad j=\overline{1, N}$,

where $G_{i, j}=G\left(\xi_{j}, x_{i}\right)$ and $G_{i, j}^{\prime}=\frac{\partial G}{\partial n}\left(\xi_{j}, x_{i}\right)$. Another $M / 4$ rows in the matrix $F$ are generated by applying the boundary condition (20) on the second electrode $\varepsilon_{2}$, namely

$$
\begin{gathered}
F_{i, j}=G_{i, j}-\frac{2 \pi}{M \ell_{2}}\left(G_{(M / 2+1), j}+G_{(M / 2+2), j}+\ldots+G_{3 M / 4, j}\right)+z_{2} G_{i, j}^{\prime}, \\
i=\overline{(M / 2+1), 3 M / 4}, \quad j=\overline{1, N} .
\end{gathered}
$$

In addition, applying the no flux boundary condition (21) results in another $M / 2$ rows given by

$$
F_{i, j}=G_{i, j}^{\prime}, \quad i=\overline{(M / 4+1), M / 2} \cup \overline{(3 M / 4+1), M}, \quad j=\overline{1, N} .
$$

To end with, the last row in the matrix $F$ obtained from the condition (22) is:

$$
F_{(M+1), j}=\sum_{i=1}^{M} G_{i, j}, \quad j=\overline{1, N}
$$


Similarly, the vector $\underline{b}$ of the linear system of equations (23) is given by

$$
\underline{b}=\left(\begin{array}{lllll}
\frac{z_{1} I_{1}}{\ell_{1}} & \underline{0} & \frac{z_{2} I_{2}}{\ell_{2}} & \underline{0} & 0
\end{array}\right)^{T} .
$$

Table 2 illustrates the numerical solutions of the problem (1), (2), (5) and (6) obtained using the MFS with various $M=N$ and $R=1.15$. From this table it can be seen that using the MFS to solve the CEM provides a convergent interior solution. However, by inspecting Tables 1 and 2 it can be seen that this convergence is slightly slower in the MFS than in the BEM, as $M=N$ increases.

Table 2: The numerical solution of Example 1 at selected interior points $(r, \theta)$ obtained using the MFS for various $M=N \in\{8,16,32,64,128,256\}$ and $R=1.15$.

\begin{tabular}{|c||c|c|c|c|c|c|}
\hline \multicolumn{1}{c|}{$\theta$} & $2 \pi / 10$ & $4 \pi / 10$ & $6 \pi / 10$ & $8 \pi / 10$ & $10 \pi / 10$ & $M$ \\
\hline \multirow{5}{*}{$1 / 10$} & 0.1316 & 0.1225 & 0.0619 & -0.0213 & -0.0968 & 8 \\
& 0.0731 & 0.0658 & 0.0334 & -0.0115 & -0.0227 & 16 \\
& 0.0578 & 0.0521 & 0.0265 & -0.0091 & -0.0413 & 32 \\
& 0.0562 & 0.0507 & 0.0257 & -0.0088 & -0.0401 & 64 \\
& 0.0562 & 0.0506 & 0.0257 & -0.0088 & -0.0401 & 128 \\
& 0.0561 & 0.0506 & 0.0257 & -0.0088 & -0.0401 & 256 \\
\hline \multirow{5}{*}{$2 / 10$} & 0.1466 & 0.1318 & 0.0664 & -0.0227 & -0.1040 & 8 \\
& 0.2740 & 0.2457 & 0.1216 & -0.0422 & -0.1909 & 16 \\
& 0.1160 & 0.1043 & 0.0526 & -0.0180 & -0.0824 & 32 \\
& 0.1127 & 0.1014 & 0.0512 & -0.0175 & -0.0801 & 64 \\
& 0.1126 & 0.1013 & 0.0511 & -0.0175 & -0.0801 & 128 \\
& 0.1126 & 0.1013 & 0.0511 & -0.0175 & -0.0800 & 256 \\
\hline \multirow{5}{*}{$3 / 10$} & 0.4144 & 0.3704 & 0.1777 & -0.0422 & -0.1909 & 8 \\
& 0.2210 & 0.1981 & 0.0982 & -0.0335 & -0.1552 & 16 \\
& 0.1747 & 0.1567 & 0.0781 & -0.0267 & -0.1230 & 32 \\
& 0.1698 & 0.1523 & 0.0759 & -0.0259 & -0.1197 & 64 \\
& 0.1697 & 0.1522 & 0.0759 & -0.0259 & -0.1196 & 128 \\
& 0.1696 & 0.1522 & 0.0759 & -0.0259 & -0.1195 & 256 \\
\hline$\ldots$ & $\ldots$ & $\ldots$ & $\ldots$ & $\ldots$ & $\ldots$ & $\ldots$ \\
\hline \multirow{3}{*}{$9 / 10$} & 1.2972 & 1.1393 & 0.4321 & -0.4131 & -0.1860 & 8 \\
& 0.9671 & 0.5922 & 0.1302 & -0.0360 & -0.3042 & 16 \\
& 0.5478 & 0.4905 & 0.1825 & -0.0572 & -0.3505 & 32 \\
& 0.5270 & 0.4783 & 0.1791 & -0.0564 & -0.3439 & 64 \\
& 0.5265 & 0.4775 & 0.1792 & -0.0564 & -0.3439 & 128 \\
& 0.5263 & 0.4773 & 0.1792 & -0.0564 & -0.3437 & 256 \\
\hline
\end{tabular}

Figures 5 and 6 show comparisons between the BEM and MFS solutions for 
the boundary data $u(1, \theta)$ and $\partial u / \partial n(1, \theta)$, respectively. In these figures the markers are shown only on a coarse selection of boundary points in order to allow the curves to be distinguishable. In the MFS, we present the results obtained with $R=1.15$ which is the optimal choice for which the numerical MFS results are the closest to the BEM results. In the absence of these latter numerical results, or of an analytical solution being available one could still optimize the choice of $R$ by minimizing (with respect to $R$ ) the error in satisfying, in a least-squares sense, the boundary conditions (2), (5) and (6) at points on the boundary different than the collocation points $\left(\underline{x}_{i}\right)_{i=\overline{1, M}}$. The reason why $R$ is close to unity is because the boundary value problem possesses singularities in the normal derivative, see Figure 5 , at the end points of electrodes where the Robin boundary condition (3) and the Neumann boundary condition (5) mix. This in turn means that the harmonic solution $u$ cannot be analytically continued too far outside the unit disk $\Omega$ and the MFS approximation (18) is accurate only provided that the sources $\left(\underline{\xi}_{j}\right)_{j=\overline{1, N}}$ are positioned on a circle of radius $R>1$ such that there are no singularities in $u$ in the circular annulus $\left\{(x, y)^{2} \in \mathbb{R}^{2} \mid 1<x^{2}+y^{2}<R^{2}\right\}$. From Figure 5 it can be seen that there is excellent agreement between the BEM and MFS numerical solutions except for the coarse meshes/degrees of freedom of 8 to 16 elements. However, increasing the number of collocation points $M$ and the degrees of freedom $N$, both $u(1, \theta)$ and its derivative $\partial u / \partial n(1, \theta)$ show good agreement with the BEM solution. Furthermore, the MFS gives the closest agreement to the BEM results with $M=N=128$ and $R=1.15$. However, for the large choice of $M=N=256$, the MFS shows some slight instability in the normal derivative, see Figure 6 . This instability is due to the ill-conditioning of the matrix $F$ which is commonly known with the MFS, see $[7,17,21]$. 

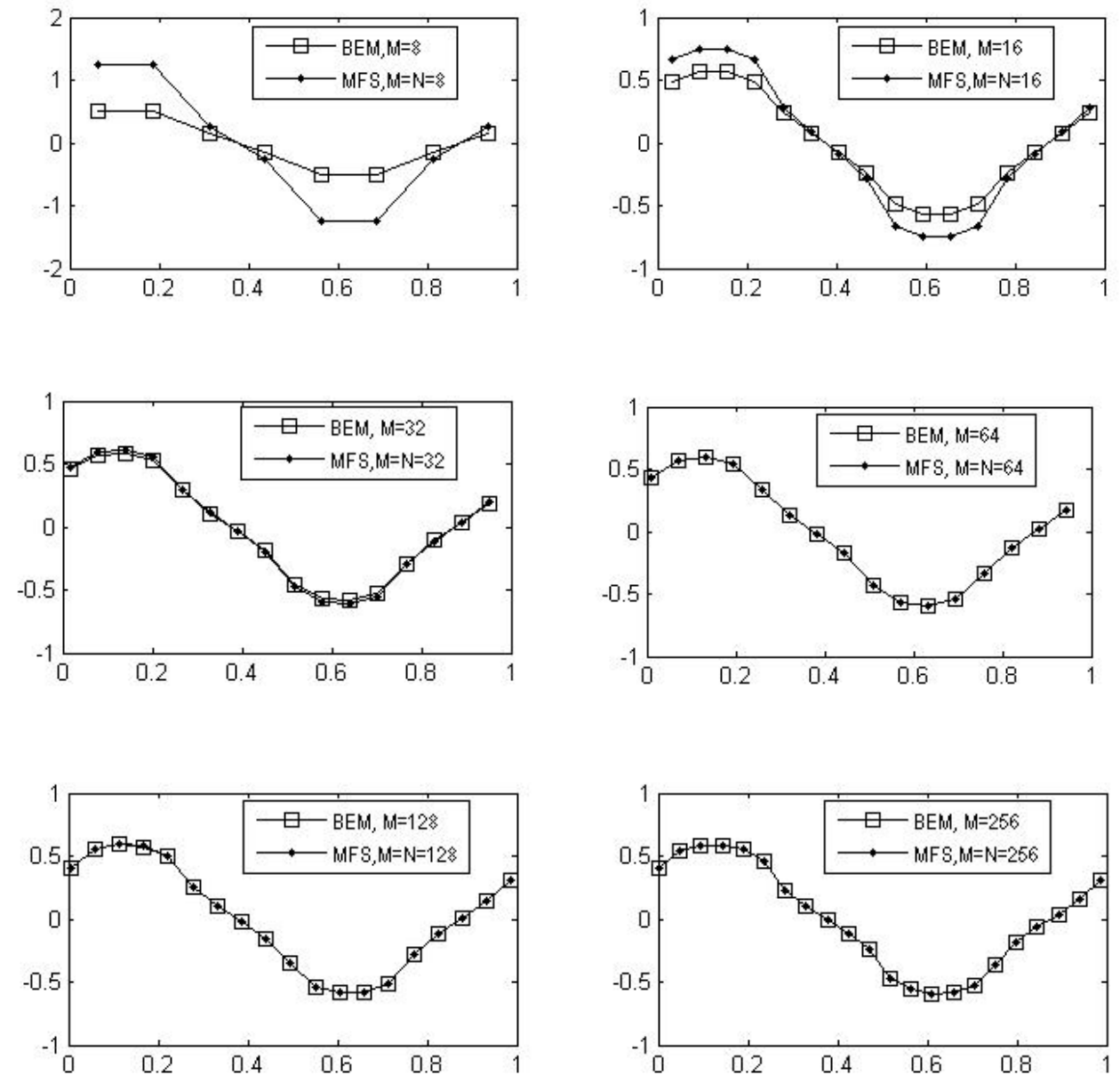

Figure 5: Comparison between $u^{M F S}(1, \theta)$ and $u^{B E M}(1, \theta)$, as functions of $\theta /(2 \pi)$, for Example 1. 



Figure 6: Comparison between $\frac{\partial u}{\partial n}^{M F S}(1, \theta)$ and $\frac{\partial u}{\partial n}^{B E M}(1, \theta)$, as functions of $\theta /(2 \pi)$, for Example 1.

Table 3 shows the condition numbers, defined as the ratio between the largest singular value to the smallest one, of the BEM and MFS matrices $D$ and $F$, respectively. This table shows that the BEM matrix $D$ is well-conditioned, but the MFS matrix $F$ is ill-conditioned. 
Table 3: Condition numbers of the matrices $D$ and $F$ of the BEM and MFS systems of equations (16) and (23), respectively, for various numbers of boundary elements $M$ (in the BEM) and degrees of freedom $M=N$ (in the MFS with $R=1.15$ ), for Example 1.

\begin{tabular}{|c||c|c|c|c|c|c|}
\hline$M=N$ & 8 & 16 & 32 & 64 & 128 & 256 \\
\hline $\operatorname{cond}(D)$ & 35.58 & 86.62 & 215.97 & 484.40 & $10^{3}$ & $2 \times 10^{3}$ \\
\hline $\operatorname{cond}(F)$ & $3 \times 10^{16}$ & $5 \times 10^{16}$ & $3 \times 10^{17}$ & $7 \times 10^{16}$ & $2 \times 10^{17}$ & $4 \times 10^{18}$ \\
\hline
\end{tabular}

Example 2. We next automate both the BEM and MFS computational codes (increasing the number of electrodes to $L=4$ and 8 ) to solve the CEM problem (1), (2), (5) and (6) with the input data $z_{p}=1$ for $p=\overline{1, L}$ and injected currents

$$
I_{p}= \begin{cases}1 & \text { if } p=1 \\ -1 & \text { if } p=L \\ 0 & \text { if } p \in\{2, \ldots, L-1\}\end{cases}
$$

Solution: If we choose $L=2$, we just re-obtain Example 1. Although the computational code of the problem (written in MATLAB) is valid for any number of electrodes $L$, only two cases will be illustrated here. These cases are $L=4$ and $L=8$.

Table 4 shows the numerical MFS and BEM interior solutions and the absolute errors between them. It can be seen that for both $L=4$ and $L=8$, the MFS and the BEM interior solutions agree up to three decimal places. In addition, the accuracy increases as we move further towards the centre of the unit disk. 
Table 4: The BEM numerical solution (with $M=128$ ) of Example 2 at the some interior points and (in brackets) the absolute errors between the BEM and MFS (with $M=N=128)$.

\begin{tabular}{|c|c|c|c|c|c|}
\hline \multicolumn{6}{|c|}{$L=4$} \\
\hline$r$ & $2 \pi / 10$ & $4 \pi / 10$ & $6 \pi / 10$ & $8 \pi / 10$ & $10 \pi / 10$ \\
\hline $1 / 10$ & $\begin{array}{c}0.0394 \\
\left(1 \times 10^{-5}\right)\end{array}$ & $\begin{array}{c}0.0426 \\
\left(1 \times 10^{-5}\right)\end{array}$ & $\begin{array}{c}0.0301 \\
\left(8 \times 10^{-6}\right)\end{array}$ & $\begin{array}{c}0.0088 \\
\left(2 \times 10^{-6}\right)\end{array}$ & $\begin{array}{c}-0.0146 \\
\left(3 \times 10^{-6}\right)\end{array}$ \\
\hline $2 / 10$ & $\begin{array}{c}0.0841 \\
\left(3 \times 10^{-5}\right)\end{array}$ & $\begin{array}{c}0.0836 \\
\left(2 \times 10^{-5}\right)\end{array}$ & $\begin{array}{c}0.0551 \\
\left(1 \times 10^{-5}\right)\end{array}$ & $\begin{array}{c}0.0156 \\
\left(3 \times 10^{-6}\right)\end{array}$ & $\begin{array}{c}-0.0259 \\
\left(5 \times 10^{-6}\right)\end{array}$ \\
\hline $3 / 10$ & $\begin{array}{c}0.1340 \\
\left(5 \times 10^{-5}\right)\end{array}$ & $\begin{array}{c}0.1223 \\
\left(3 \times 10^{-5}\right)\end{array}$ & $\begin{array}{c}0.0752 \\
\left(1 \times 10^{-5}\right)\end{array}$ & $\begin{array}{c}0.0207 \\
\left(3 \times 10^{-6}\right)\end{array}$ & $\begin{array}{c}-0.0345 \\
\left(5 \times 10^{-6}\right)\end{array}$ \\
\hline$\cdots$ & $\cdots$ & $\cdots$ & $\cdots$ & $\cdots$ & $\cdots$ \\
\hline $9 / 10$ & $\begin{array}{c}0.5723 \\
\left(4 \times 10^{-4}\right)\end{array}$ & $\begin{array}{c}0.2593 \\
\left(3 \times 10^{-5}\right)\end{array}$ & $\begin{array}{c}0.1216 \\
\left(3 \times 10^{-5}\right)\end{array}$ & $\begin{array}{c}0.0330 \\
\left(3 \times 10^{-6}\right)\end{array}$ & $\begin{array}{c}-0.0560 \\
\left(8 \times 10^{-6}\right)\end{array}$ \\
\hline \multicolumn{6}{|c|}{$L=8$} \\
\hline I & $2 \pi / 10$ & $4 \pi / 10$ & $6 \pi / 10$ & $8 \pi / 10$ & $10 \pi / 10$ \\
\hline $1 / 10$ & $\begin{array}{c}0.0199 \\
\left(7 \times 10^{-6}\right)\end{array}$ & $\begin{array}{c}0.0242 \\
\left(7 \times 10^{-6}\right) \\
\end{array}$ & $\begin{array}{c}0.0191 \\
\left(4 \times 10^{-6}\right)\end{array}$ & $\begin{array}{c}0.0085 \\
\left(2 \times 10^{-6}\right) \\
\end{array}$ & $\begin{array}{c}-0.0039 \\
\left(9 \times 10^{-7}\right)\end{array}$ \\
\hline $2 / 10$ & $\begin{array}{c}0.0449 \\
\left(1 \times 10^{-5}\right)\end{array}$ & $\begin{array}{c}0.0484 \\
\left(1 \times 10^{-5}\right)\end{array}$ & $\begin{array}{c}0.0347 \\
\left(7 \times 10^{-6}\right)\end{array}$ & $\begin{array}{c}0.0147 \\
\left(2 \times 10^{-6}\right)\end{array}$ & $\begin{array}{c}-0.0067 \\
\left(1 \times 10^{-6}\right)\end{array}$ \\
\hline $3 / 10$ & $\begin{array}{c}0.0752 \\
\left(2 \times 10^{-5}\right)\end{array}$ & $\begin{array}{c}0.0714 \\
\left(1 \times 10^{-5}\right)\end{array}$ & $\begin{array}{c}0.0469 \\
\left(7 \times 10^{-6}\right)\end{array}$ & $\begin{array}{c}0.0191 \\
\left(2 \times 10^{-6}\right)\end{array}$ & $\begin{array}{c}-0.0087 \\
\left(1 \times 10^{-6}\right)\end{array}$ \\
\hline$\cdots$ & $\cdots$ & $\cdots$ & $\cdots$ & $\cdots$ & $\cdots$ \\
\hline $9 / 10$ & $\begin{array}{c}0.3099 \\
\left(1 \times 10^{-4}\right)\end{array}$ & $\begin{array}{c}0.1451 \\
\left(1 \times 10^{-5}\right)\end{array}$ & $\begin{array}{c}0.0748 \\
\left(4 \times 10^{-6}\right)\end{array}$ & $\begin{array}{c}0.0276 \\
\left(4 \times 10^{-7}\right)\end{array}$ & $\begin{array}{c}-0.0127 \\
\left(2 \times 10^{-6}\right)\end{array}$ \\
\hline
\end{tabular}

Figures 7 and 8 represent the comparison on the boundary for $L=4$ and 8 , respectively. From these figures it can be seen that both methods still follow the same pattern as for the case $L=2$. 

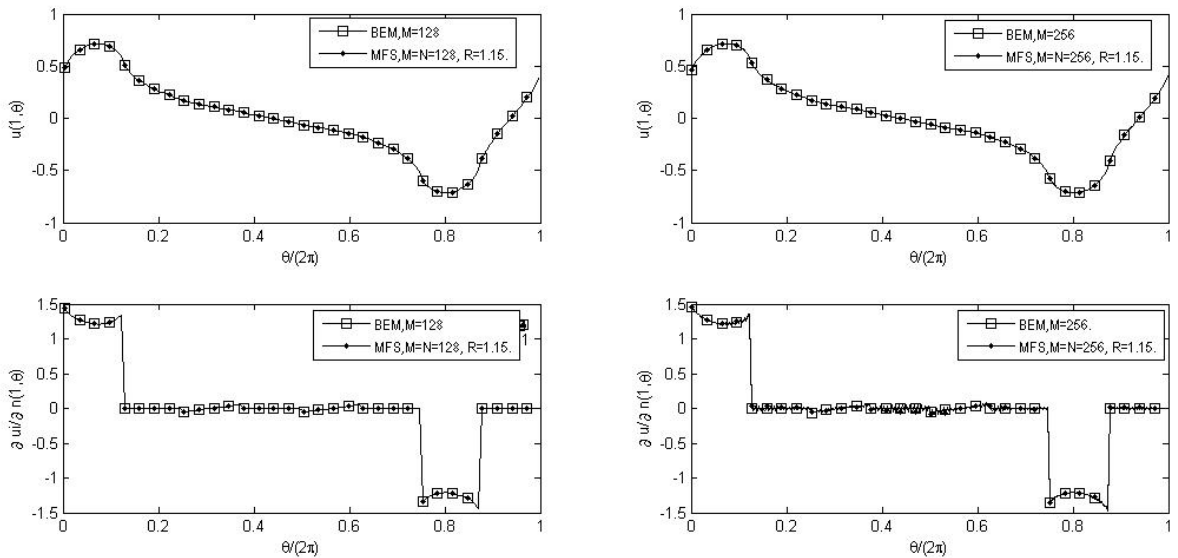

Figure 7: Comparison between the MFS and BEM solutions and their normal derivatives on the boundary when the number of electrodes is $L=4$.
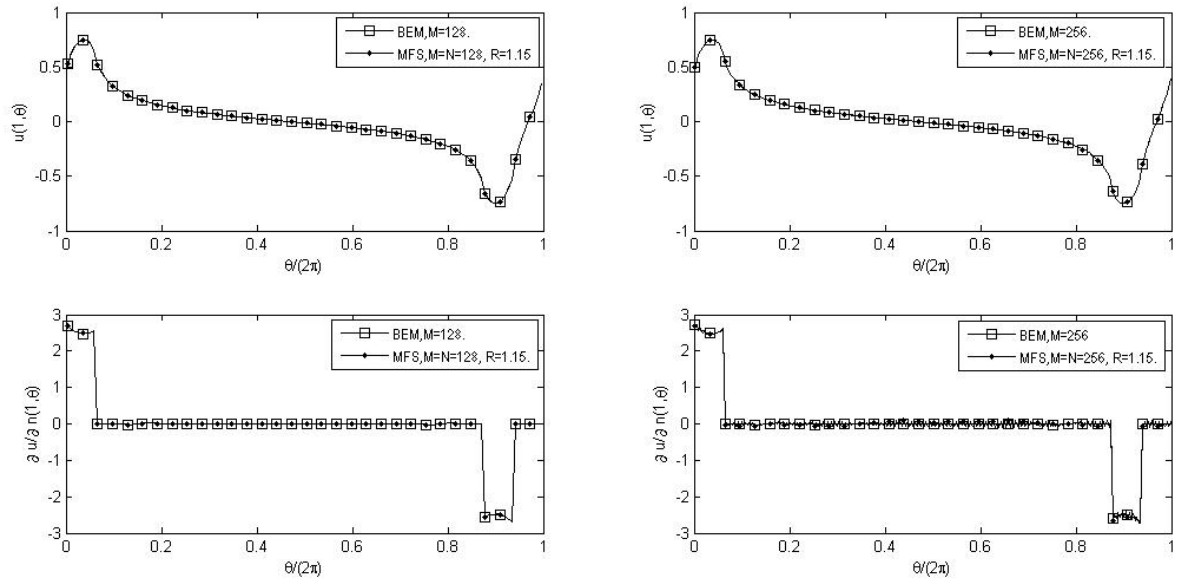

Figure 8: Comparison between the MFS and BEM solutions and their normal derivatives on the boundary when the number of electrodes is $L=8$.

\section{Extension to Multiply-Connected Domains}

So far, the solution domain $\Omega$ (unit disk), which has been considered, was a simplyconnected domain. In this section, we will investigate the direct EIT problem in a domain which has a void (rigid inclusion or cavity) inside. 


\subsection{Applying the BEM to the Direct EIT Problem in an Annular Domain with a Rigid Inclusion}

Here, the solution domain $\Omega$ is the annulus $\left\{(x, y) \in \mathbb{R}^{2} \mid(0.5)^{2}<x^{2}+y^{2}<1\right\}$, where on the boundary of the hole inside (rigid inclusion), the boundary condition is $u=0$.

First, the external boundary $r=1$ is uniformly discretised into $M$ boundary elements and the numbering of these elements is anticlockwise. Similarly, the internal boundary $r=0.5$ is uniformly discretized into another $M$ boundary elements, but these are numbered clockwise, [22]. The endpoints of external boundary elements are

$$
\underline{p_{i}}=\left(x_{i}, y_{i}\right)=\left(\cos \left(\frac{2 \pi i}{M}\right), \sin \left(\frac{2 \pi i}{M}\right)\right), \quad i=\overline{1, M},
$$

with the convention that $\underline{p}_{0}=\underline{p}_{M}$, whereas the endpoints of internal boundary elements are

$$
\begin{array}{r}
\underline{p_{i}}=\left(x_{i}, y_{i}\right)=\left(0.5 \cos \left(2 \pi-\frac{2 \pi(i-M)}{M}\right), 0.5 \sin \left(2 \pi-\frac{2 \pi(i-M)}{M}\right)\right), \\
i=\frac{M+1,2 M}{M} .
\end{array}
$$

Since $u=0$ on the boundary of the inner rigid inclusion, the EIT problem is reduced to solving a new linear system of BEM equations

$$
B \underline{u}_{O u t e r}+A \underline{u^{\prime}}=\underline{0},
$$

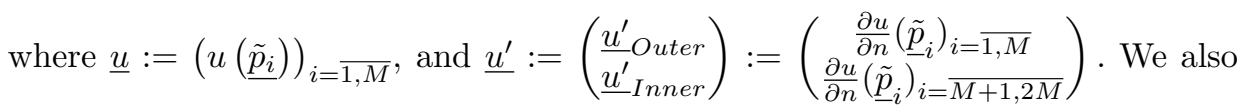
denote the boundary element node $\tilde{p}_{i}=\left(p_{i}+p_{i-1}\right) / 2$ for $i=\overline{1, M} \cup \overline{M+2,2 M}$, and $\tilde{p}_{M+1}=\left(p_{M+1}+p_{2 M}\right) / 2$.

First, we collocate the boundary condition (2) for the electrodes $\varepsilon_{p}, p=\overline{1, L}$, at the nodes $\tilde{p}_{i-2 M}$, resulting in

$$
\begin{aligned}
u_{i-2 M}+z_{p} u_{i-2 M}^{\prime}- & \frac{2 \pi}{M \ell_{p}} \sum_{k=(K M / L)+1}^{(2 K+1) M /(2 L)} u_{k}=\frac{z_{p} I_{p}}{\ell_{p}}, \\
i & =\overline{(2 M+1+K M / L),(2 M+(2 K+1) M /(2 L)},
\end{aligned}
$$

where $K=\overline{0,(L-1)}$. This yields $M / 2$ equations.

Second, by applying the zero flux boundary condition (5) for the gaps $g_{p}$, $p=\overline{1, L}$, between electrodes at the nodes $\tilde{p}_{i-2 M}$, we obtain

$$
u_{i-2 M}^{\prime}=0, \quad i=\overline{(2 M+1+(2 K-1) M /(2 L)),(2 M+K M / L)},
$$


where $K=\overline{1, L}$. This yields another $M / 2$ equations.

Finally, the condition

$$
\int_{\partial \Omega_{\text {outer }}} u d s=0
$$

yields one more equation, namely,

$$
\sum_{k=1}^{M} u_{k}=0
$$

Therefore, to find the solution of the CEM problem (1), (2), (5) and (6) in an annular domain containing an inner rigid inclusion using the BEM, the equations (27)-(29) are reformulated in the following matrix form as a $(3 M+1) \times(3 M)$ linear system of algebraic equations:

$$
D \underline{X}=\underline{b}
$$

where

$$
\underline{X}=\left(\begin{array}{l}
\underline{u}_{\text {Outer }} \\
\underline{u}_{\text {Outer }}^{\prime} \\
\underline{u}_{\text {Inner }}^{\prime}
\end{array}\right) .
$$

Since the system of equations (30) is over-determined, we have used the leastsquares method to solve it. This yields the solution (17) for the unspecified boundary data (31).

Once the boundary data has been obtained accurately, equation (11) can be applied for $\underline{p} \in \Omega$ to provide explicitly the interior solution for $u(\underline{p})$.

\subsection{Applying the MFS to the Direct EIT Problem in an Annular Domain with a Rigid Inclusion}

In this section, the MFS seeks a solution of Laplace's equation (1) as a linear combination of fundamental solutions of the form:

$$
u(\underline{p})=\sum_{j=1}^{2 N} c_{j} G\left(\underline{\xi}_{j}, \underline{p}\right), \quad \underline{p} \in \bar{\Omega} .
$$

where $\underline{\xi}_{j}$ are the sources and located outside the outer domain

$$
\Omega_{\text {Outer }}=\left\{(x, y) \in \mathbb{R}^{2} \mid x^{2}+y^{2}=1\right\}
$$

and inside the rigid inclusion

$$
\Omega_{\text {Inner }}=\left\{(x, y) \in \mathbb{R}^{2} \mid x^{2}+y^{2}=(0.5)^{2}\right\},
$$


where $\Omega=\Omega_{\text {Outer }} \backslash \bar{\Omega}_{\text {Inner }}$. The $\left(c_{j}\right)_{j=\overline{1,2 N}}$ are unknown coefficients to be determined by imposing the boundary conditions (2), (5), (6) and

$$
u=0 \quad \text { on } \quad \partial \Omega_{\text {Inner }} .
$$

Since $\Omega=\Omega_{\text {Outer }} \backslash \bar{\Omega}_{\text {Inner }}=B(\mathbf{0} ; 1) \backslash B(\mathbf{0} ; 0.5)$, we take the external source points $\underline{\xi}_{j}=\left(\xi_{j}^{1}, \xi_{j}^{2}\right)=\left(R \cos \left(\frac{2 \pi j}{N}\right), R \sin \left(\frac{2 \pi j}{N}\right)\right)$ for $j=\overline{1, N}$, where $1<R<\infty$, the internal source points $\underline{\xi}_{j}=\left(\xi_{j}^{1}, \xi_{j}^{2}\right)=\left(R_{1} \cos \left(\frac{2 \pi(j-N)}{N}\right), R_{1} \sin \left(\frac{2 \pi(j-N)}{N}\right)\right)$, for $j=\overline{N+1,2 N}$, where $0<R_{1}<0.5$. We also take the external boundary collocation points $\underline{x}_{i}=\left(\cos \left(\frac{2 \pi i}{M}\right), \sin \left(\frac{2 \pi i}{M}\right)\right)$ for $i=\overline{1, M}$, and the internal boundary collocation points $\underline{x}_{i}=\left(0.5 \cos \left(\frac{2 \pi(j-M)}{M}\right), 0.5 \sin \left(\frac{2 \pi(j-M)}{M}\right)\right)$ for $i=\overline{M+1,2 M}$.

For external points $p=(x, y) \in \partial \Omega_{\text {Outer }}$ we have

$$
\frac{\partial G}{\partial n}\left(\underline{\xi}_{j}, \underline{p}\right)=\frac{1-\left(\xi_{j}^{1} x+\xi_{j}^{2} y\right)}{-2 \pi\left|\underline{\xi}_{j}-\underline{p}\right|^{2}}, \quad j=\overline{1,2 N},
$$

whilst for internal points $p=(x, y) \in \partial \Omega_{\text {Inner }}$ we have

$$
\frac{\partial G}{\partial n}\left(\underline{\xi}_{j}, \underline{p}\right)=\frac{(0.5)^{2}-\left(\xi_{j}^{1} x+\xi_{j}^{2} y\right)}{-2(0.5) \pi\left|\underline{\xi}_{j}-\underline{p}\right|^{2}}, \quad j=\overline{1,2 N} .
$$

In order to obtain the coefficient vector $\underline{c}=\left(c_{j}\right)_{j=\overline{1,2 N}}$, we substitute equations (9), (34), and (35) into the boundary conditions (2), (5), (6) and (33).

First, we apply the boundary condition (2) for the electrodes $\varepsilon_{p}, p=\overline{1, L}$, at the collocation points $\underline{x}_{i}$ on $\varepsilon_{p}$ resulting in

$$
\begin{array}{r}
\sum_{j=1}^{2 N}\left[G\left(\underline{\xi}_{j}, \underline{x}_{i}\right)-\frac{2 \pi}{M \ell_{p}} \sum_{k=(K M / L)+1}^{(2 K+1) M /(2 L)} G\left(\underline{\xi}_{j}, \underline{x}_{k}\right)+z_{p} \frac{\partial G}{\partial r}\left(\underline{\xi}_{j}, \underline{x}_{i}\right)\right] c_{j}=\frac{z_{p} I_{p}}{\ell_{p}} \\
i=\overline{(K M / L)+1,(2 K+1) M /(2 L)},
\end{array}
$$

where $K=\overline{0,(L-1)}$. This yields $M / 2$ equations.

Second, by applying the zero flux boundary condition (5) on the gaps $g_{p}, p=$ $\overline{1, L}$, between electrodes, we obtain

$$
\sum_{j=1}^{2 N} c_{j} \frac{\partial G}{\partial r}\left(\underline{\xi}_{j}, \underline{x}_{i}\right)=0, \quad i=\overline{(1+(2 K-1) M /(2 L)),(K M / L)} .
$$

where $K=\overline{1, L}$. This yields another $M / 2$ equations.

Third, we apply (33) which gives $M$ more equations

$$
\sum_{j=1}^{2 N} c_{j} G\left(\xi_{j}, \underline{x}_{i}\right)=0, \quad i=\overline{M+1,2 M} .
$$


Finally, by imposing the condition (6) and using (38), yields one more equation

$$
\sum_{i=1}^{2 M} \sum_{j=1}^{2 N} c_{j} G\left(\underline{\xi}_{j}, \underline{x}_{i}\right)=0 .
$$

Again, to find the solution of the CEM problem (1), (2), (5), (6) and (33) using the MFS, the equations (36)-(39) are reformulated in the following matrix form as a $(2 M+1) \times 2 N$ linear system of algebraic equations:

$$
F \underline{c}=\underline{b} .
$$

The least-squares method is used to solve the system of equations (40). This yields the solution (24).

Once the coefficient vector $\underline{c}$ has been obtained accurately, equations (32), (34) and (35) provide explicitly the solution for the potential $u_{O u t e r}$ on the external boundary $\partial \Omega_{\text {Outer }}$ and inside the annular domain $\Omega$, the current flux $(\partial u / \partial n)_{\text {Outer }}$ on the external boundary $\partial \Omega_{\text {Outer }}$ and the current flux $(\partial u / \partial n)_{\text {Inner }}$ on the internal boundary $\partial \Omega_{\text {Inner }}$, respectively.

Example 3. Solve the problem (1), (2), (5), (6) and (33) using the BEM and MFS with the same input data as in Example 1.

BEM solution: The matrix $D$ in equation (30) is given by

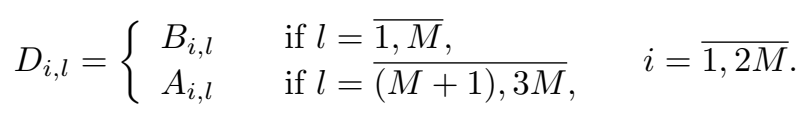

On the other hand, using equations (27)-(29) we obtain:

$$
\begin{aligned}
& D_{i, l}= \begin{cases}-\frac{h}{\ell_{1}} & \text { if }(i-2 M) \neq l, l=\overline{1, M / 4}, \\
\left(1-\frac{h}{\ell_{1}}\right) & \text { if }(i-2 M)=l, l=\overline{1, M / 4}, \\
0 & \text { if } l=\overline{(M / 4+1), M} \cup \overline{(2 M+1), 3 M}, \\
z_{1} \delta_{i, l} & \text { if } l=\overline{(M+1), 2 M},\end{cases} \\
& i=\overline{(2 M+1),(2 M+M / 4)}, \\
& D_{i, l}= \begin{cases}-\frac{h}{\ell_{2}} & \text { if }(i-2 M) \neq l, l=\overline{(M / 2+1), 3 M / 4} \\
\left(1-\frac{h}{\ell_{2}}\right) & \text { if }(i-2 M)=l, l=\overline{(M / 2+1), 3 M / 4} \\
0 & \text { if } l=\overline{1, M / 2} \cup \overline{(3 M / 4+1), M} \cup \overline{(2 M+1), 3 M} \\
z_{2} \delta_{i, l} & \text { if } l=\overline{(M+1), 2 M},\end{cases} \\
& i=\overline{(2 M+M / 2+1),(2 M+3 M / 4)},
\end{aligned}
$$




$$
\begin{gathered}
D_{i, l}=\delta_{i, l}, \quad \text { if } l=\overline{(M+1), 2 M}, \\
i=\overline{(2 M+M / 4+1),(M+M / 2)} \cup \overline{(2 M+3 M / 4+1), 3 M} .
\end{gathered}
$$

The last row in the matrix $D$ is given by

$$
D_{(3 M+1), l}= \begin{cases}1 & \text { if } l=\overline{1, M} \\ 0 & \text { if } l=\overline{M+1,3 M} .\end{cases}
$$

Furthermore, the vector $\underline{b}$ is given by

$$
\underline{b}=\left(\begin{array}{llllll}
\underline{0} & \frac{z_{1} I_{1}}{\ell_{1}} & \underline{0} & \frac{z_{2} I_{2}}{\ell_{2}} & \underline{0} & 0
\end{array}\right)^{T} .
$$

MFS solution: Turning now to the MFS solution, the first $M / 4$ rows of the matrix $F$ in equation (40) coresponding to the first electrode $\varepsilon_{1}$ are

$F_{i, j}=G_{i, j}-\frac{2 \pi}{M \ell_{1}}\left(G_{i, j}+G_{i+1, j}+\ldots+G_{M / 4, j}\right)+z_{1} G_{i, j}^{\prime}, \quad i=\overline{1, M / 4}, \quad j=\overline{1,2 N}$.

Another $M / 4$ rows in the matrix $F$ are generated by applying the boundary condition (2) on the second electrode $\varepsilon_{2}$, namely

$$
\begin{gathered}
F_{i, j}=G_{i, j}-\frac{2 \pi}{M \ell_{2}}\left(G_{(M / 2+1), j}+G_{(M / 2+2), j}+\ldots+G_{3 M / 4, j}\right)+z_{2} G_{i, j}^{\prime}, \\
i=\overline{(M / 2+1), 3 M / 4}, \quad j=\overline{1,2 N} .
\end{gathered}
$$

In addition, applying the no flux boundary condition (5) results in another $M / 2$ rows given by

$$
F_{i, j}=G_{i, j}^{\prime}, \quad i=\overline{(M / 4+1), M / 2} \cup \overline{3 M / 4+1}, \quad j=\overline{1,2 N} .
$$

Moreover, another $M$ rows are generated from applying the inner boundary condition (33), namely,

$$
F_{i, j}=G_{i, j}, \quad i=\overline{(M+1), 2 M}, \quad j=\overline{1,2 N} .
$$

To end with, the last row in the matrix $F$ obtained using equation (39) is:

$$
F_{(M+1), j}=\sum_{i=1}^{2 M} G_{i, j}, \quad j=\overline{1,2 N}
$$


The vector $\underline{b}$ of the linear system of equations (40) is given by

$$
\underline{b}=\left(\begin{array}{lllll}
\frac{z_{1} I_{1}}{\ell_{1}} & \underline{0} & \frac{z_{2} I_{2}}{\ell_{2}} & \underline{0} & 0
\end{array}\right)^{T} .
$$

In the MFS we take $R=1.15$ and $R_{1}=0.45$. Figures 9,10 and 11 present a comparison between the BEM and MFS solutions for the boundary data $u_{\text {Outer }}(1, \theta)$, $(\partial u / \partial n)_{\text {Outer }}(1, \theta)$ and $(\partial u / \partial n)_{\text {Inner }}(0.5, \theta)$, respectively. From these figures it can be seen that the BEM outer solution and its derivative, as well as the BEM inner derivative are convergent, as the number of boundary elements $M$ increases. This is also true when the MFS is used except for $M=N=256$, where the outer solution still has a reasonable accuracy, but the normal derivative on the inner boundary becomes highly unstable, see also Table 5 for the condition numbers.
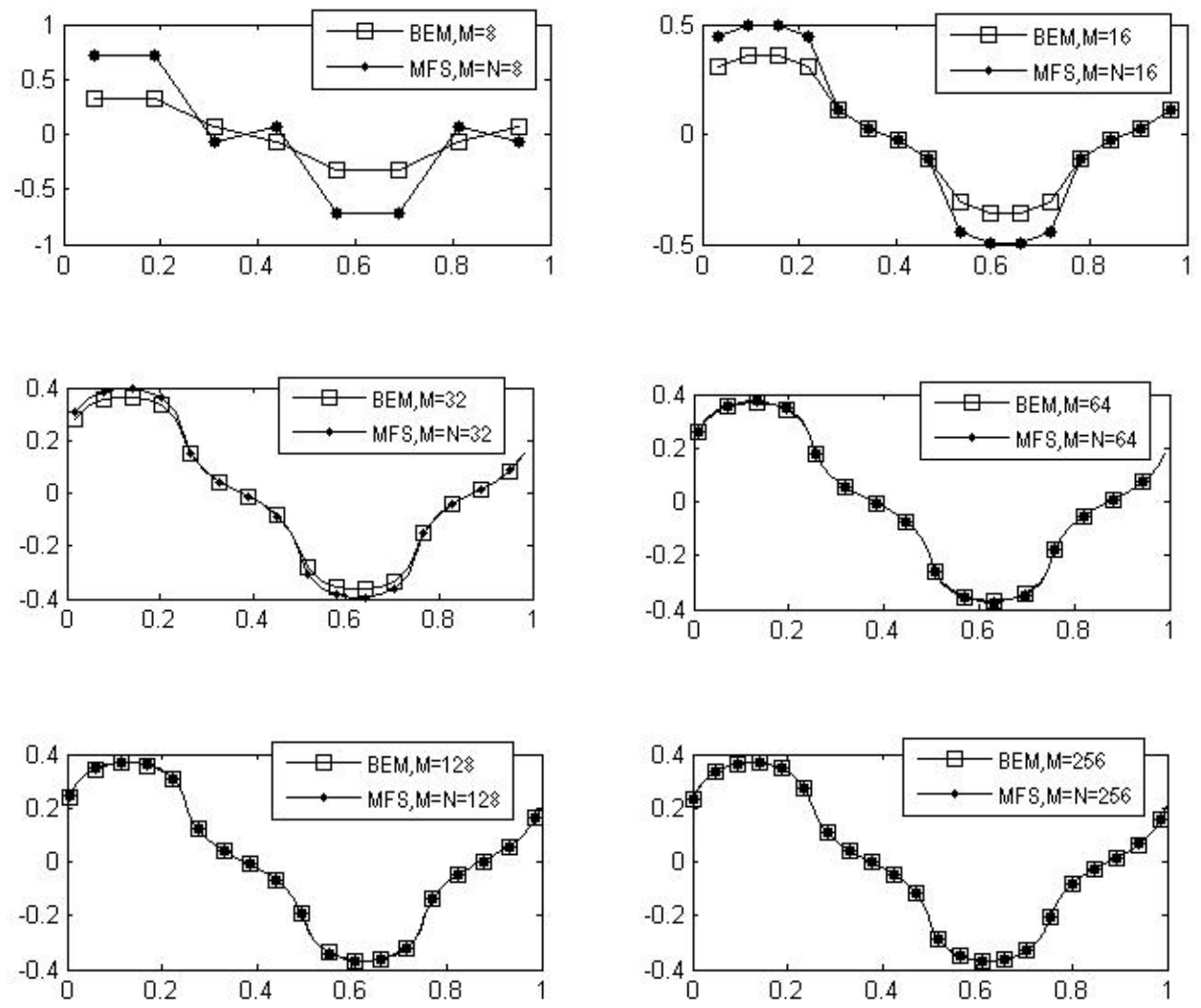

Figure 9: Comparison between $u_{\text {Outer }}^{M F S}(1, \theta)$ and $u_{\text {Outer }}^{B E M}(1, \theta)$, as functions of $\theta /(2 \pi)$, for Example 3. 

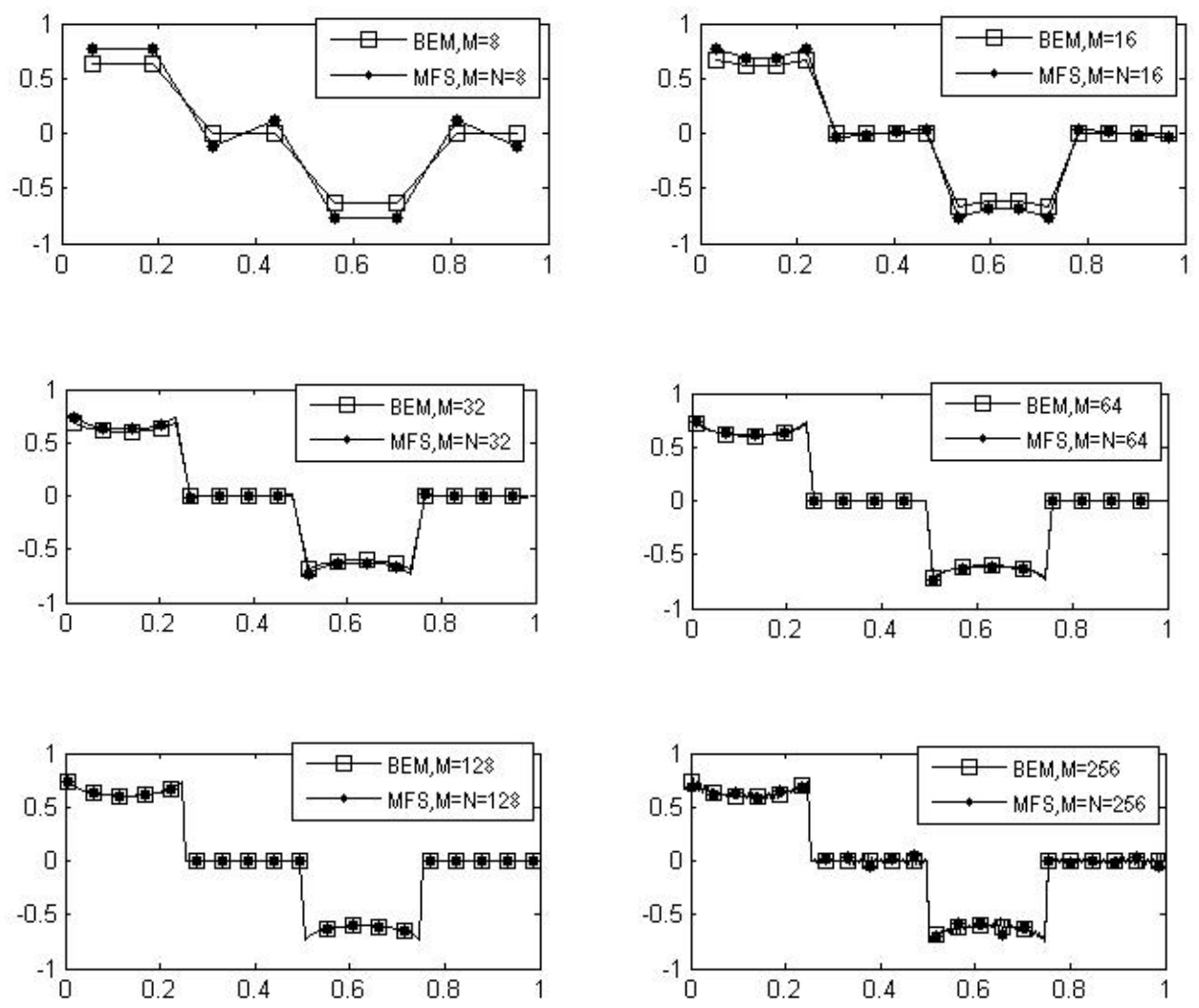

Figure 10: Comparison between $\left(\frac{\partial u}{\partial n}\right)_{\text {Outer }}^{M F S}(1, \theta)$ and $\left(\frac{\partial u}{\partial n}\right)_{\text {Outer }}^{B E M}(1, \theta)$, as functions of $\theta /(2 \pi)$, for Example 3 . 

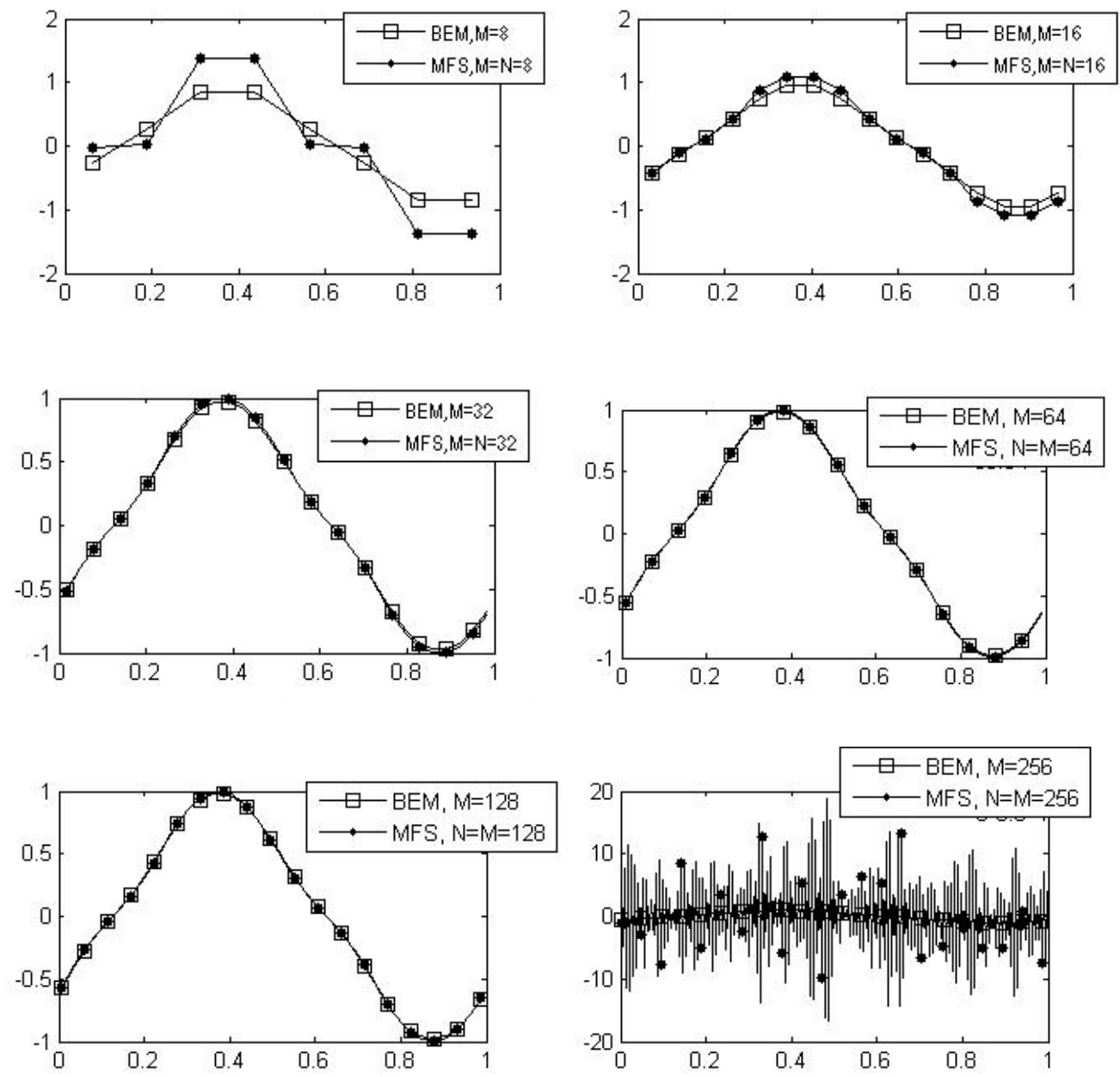

Figure 11: Comparison between $\left(\frac{\partial u}{\partial n}\right)_{\text {Inner }}^{M F S}(0.5, \theta)$ and $\left(\frac{\partial u}{\partial n}\right)_{\text {Inner }}^{B E M}(0.5, \theta)$, as functions of $\theta /(2 \pi)$, for Example 3 . 
Table 5: Condition numbers of the matrices $D$ and $F$ of the BEM and MFS systems of equations (30) and (40), respectively, for various numbers of boundary elements $M$ (in the BEM) and degrees of freedom $M=N$ (in the MFS with $R=1.15$ and $R_{1}=0.45$ ), for Example 3.

\begin{tabular}{|c||c|c|c|c|c|c|}
\hline & 8 & 16 & 32 & 64 & 128 & 256 \\
\hline $\operatorname{cond}(D)$ & $2 \times 10^{3}$ & $10^{4}$ & $10^{5}$ & $7 \times 10^{5}$ & $6 \times 10^{6}$ & $5 \times 10^{7}$ \\
\hline $\operatorname{cond}(F)$ & $10^{17}$ & $6 \times 10^{18}$ & $2 \times 10^{18}$ & $5 \times 10^{18}$ & $4 \times 10^{17}$ & $2 \times 10^{19}$ \\
\hline
\end{tabular}

\subsection{Applying the BEM to the Direct EIT Problem in an Annular Domain with a Cavity}

Herein, the solution domain $\Omega$ is the same annulus as in Section 6.1, but now it contains a cavity inside on whose boundary $\partial u / \partial n=0$.

The BEM implementation is the same as that for the rigid inclusion of Section 6.1 , however now the BEM reduces to solving the system of equations

$$
B \underline{u}+A \underline{u}_{O u t e r}^{\prime}=\underline{0},
$$

where $\underline{u}:=\left(\begin{array}{c}\underline{u}_{\text {Outer }} \\ \underline{u}_{\text {Inner }}\end{array}\right):=\left(\begin{array}{c}u\left(\underline{\tilde{p}}_{i}\right)_{i=\overline{1, M}} \\ u\left(\underline{\tilde{p}}_{i}\right)_{i=\overline{M+1,2 M}}\end{array}\right)$, and $\underline{u}_{\text {Outer }}:=\left(\frac{\partial u}{\partial n}\left(\underline{\tilde{p}}_{i}\right)\right)_{i=\overline{1, M}}$.

Equations (27)-(29) remain the same. Therefore, to find the solution of the CEM (1), (2), (5) and (6) in an annular domain with a cavity using the BEM, the equations (27)-(29) and (43) are reformulated in the following matrix form as a $(3 M+1) \times(3 M)$ linear system of algebraic equations:

$$
D \underline{X}=\underline{b},
$$

where

$$
\underline{X}=\left(\begin{array}{l}
\underline{u}_{\text {Outer }} \\
\underline{u}_{\text {Outer }}^{\prime} \\
\underline{u}_{\text {Inner }}
\end{array}\right) .
$$

Since the system of equations (44) is over-determined, we have used the leastsquares method to solve it. This yields the solution (17) for the unspecified boundary data (45). Afterwards, equation (11) can be applied for $\underline{p} \in \Omega$ to provide explicitly the interior solution for $u(\underline{p})$.

\subsection{Applying the MFS to the Direct EIT Problem in an Annular Domain with a Cavity}

Using the MFS to solve the forward EIT problem in a region which contains a cavity inside is similar to solving the problem with the rigid inclusion of section 6.2. 
The only difference is the the internal Dirichlet homogenous boundary condition (33) is replaced by the zero flux boundary condition

$$
\frac{\partial u}{\partial n}=0 \text { on } \partial \Omega_{\text {Inner }} .
$$

Hence,

$$
\sum_{j=1}^{2 N} c_{j} \frac{\partial G}{\partial n}\left(\xi_{j}, \underline{x}_{i}\right)=0, \quad i=\overline{M+1,2 M} .
$$

Due to this change, the rows

$$
F_{i, j}=G_{i, j}^{\prime}, \quad i=\overline{(M+1), 2 M}, \quad j=\overline{1,2 N},
$$

will be updated in the new matrix $F$.

Example 4. Solve the problem (1), (2), (5), (6) and (46) using the BEM and MFS with the same input data as in Example 1.

Solution: The matrix $D$ in equation (44) has the same structure as for Example 3 , but the last row is given by

$$
D_{(3 M+1), l}= \begin{cases}1 & \text { if } l=\overline{1, M} \cup \overline{2 M+1,3 M} \\ 0 & \text { if } l=\overline{M+1,2 M}\end{cases}
$$

Furthermore, the vector $\underline{b}$ is the same as that given by (41).

Figures 12,13 and 14 present a comparison between the BEM and MFS solution for the boundary data $u_{\text {Outer }}(1, \theta)$ and $(\partial u / \partial n)_{\text {Outer }}(1, \theta)$ and $u_{\text {Inner }}(0.5, \theta)$, respectively. First, from Figures 12 and 13 the same conclusions, as those obtained from Figures 9 and 10 for the rigid inclusion problem of Example 3, can be drawn for the cavity problem of Example 4. Second, for the large $M=N=256$, the MFS instability manifested in retrieving the normal derivative on the inner boundary of the rigid inclusion highlighted in Figure 11 is not present in Figure 14 which shows the function $u$ on the inner boundary of the cavity. The reason for this is that retrieving higher order derivatives is less accurate and less stable than retrieving lower order ones, [19]. 

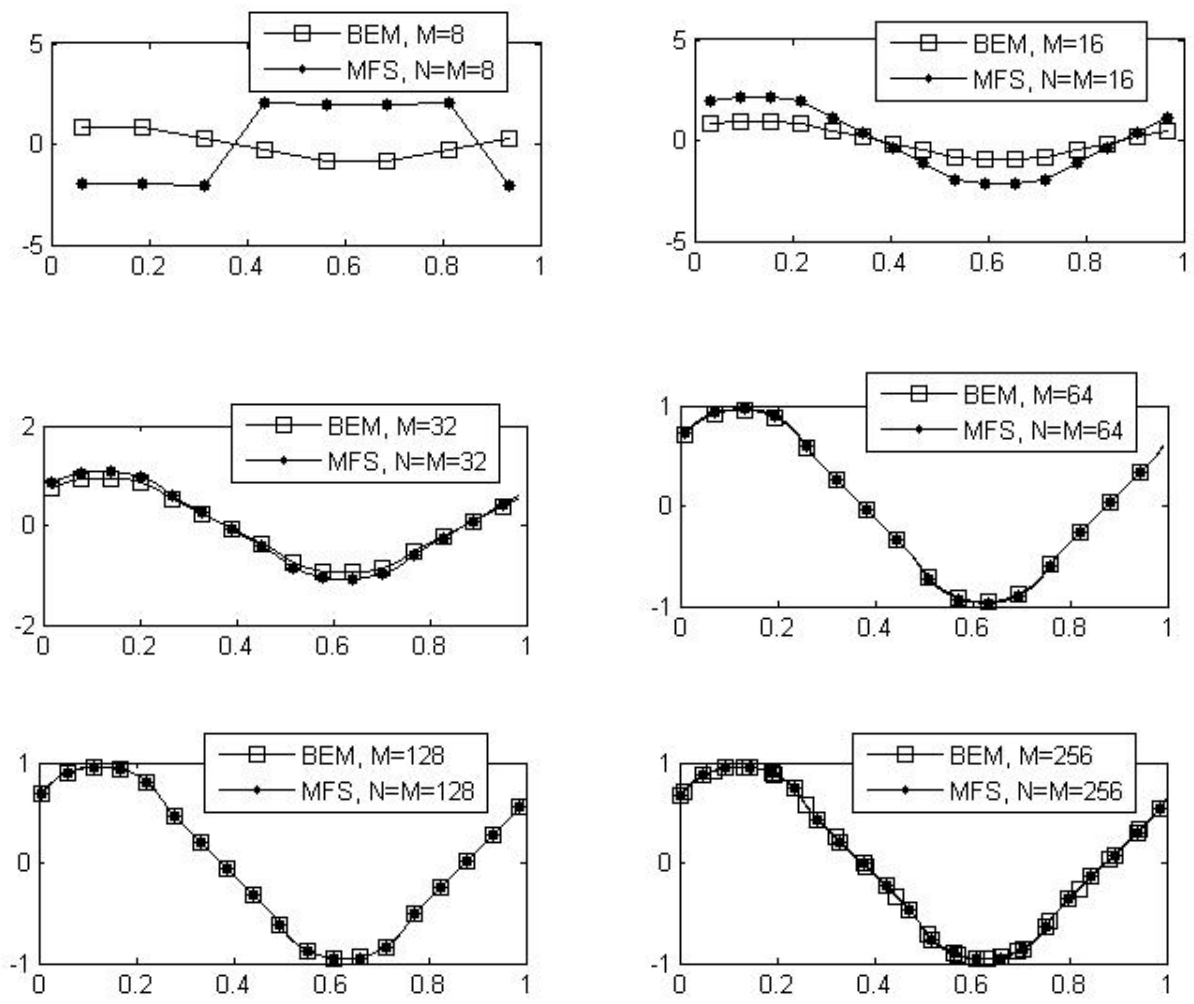

Figure 12: Comparison between $u_{\text {Outer }}^{M F S}(1, \theta)$ and $u_{\text {Outer }}^{B E M}(1, \theta)$, as functions of $\theta /(2 \pi)$, for Example 4. 



Figure 13: Comparison between $\left(\frac{\partial u}{\partial n}\right)_{\text {Outer }}^{M F S}(1, \theta)$ and $\left(\frac{\partial u}{\partial n}\right)_{\text {Outer }}^{B E M}(1, \theta)$, as functions of $\theta /(2 \pi)$, for Example 4 . 

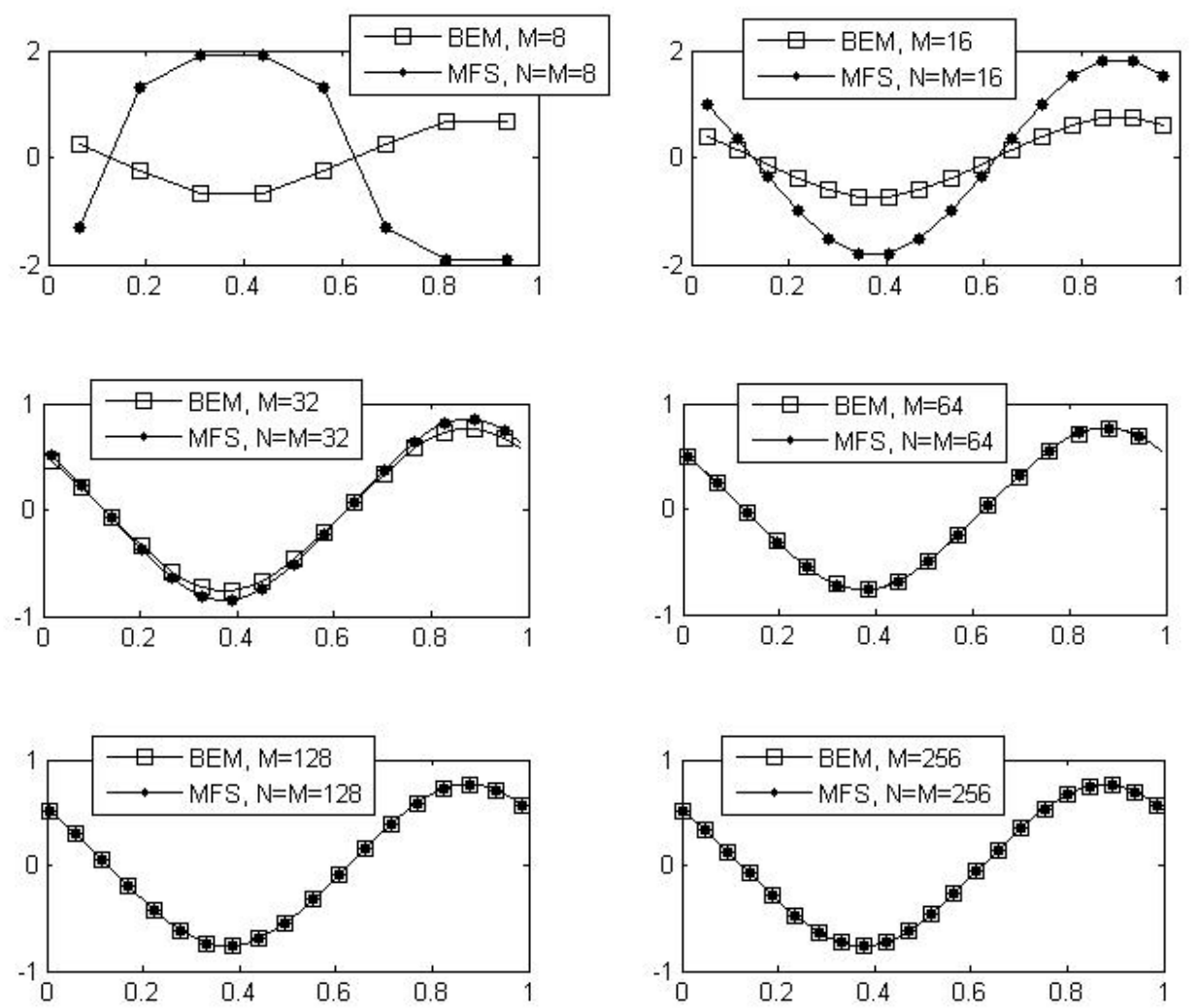

Figure 14: Comparison between $u_{\text {Inner }}^{M F S}(0.5, \theta)$ and $u_{\text {Inner }}^{B E M}(0.5, \theta)$, as functions of $\theta /(2 \pi)$, for Example 4.

Table 6 shows the condition numbers of the BEM and MFS matrices $D$ and $F$, respectively. This table shows that the BEM matrix $D$ is well-conditioned, but the MFS matrix $F$ is ill-conditioned.

Table 6: Condition numbers of the matrices $D$ and $F$ of the BEM and MFS systems of equations, for various numbers of boundary elements $M$ (in the BEM) and degrees of freedom $M=N$ (in the MFS with $R=1.15$ and $R_{1}=0.45$ ), for Example 4 .

\begin{tabular}{|c||c|c|c|c|c|c|}
\hline & 8 & 16 & 32 & 64 & 128 & 256 \\
\hline $\operatorname{cond}(D)$ & 311.91 & 643.53 & $10^{3}$ & $2 \times 10^{3}$ & $5 \times 10^{3}$ & $\times 10^{4}$ \\
\hline $\operatorname{cond}(F)$ & $2 \times 10^{17}$ & $5 \times 10^{16}$ & $10^{18}$ & $9 \times 10^{17}$ & $5 \times 10^{17}$ & $10^{18}$ \\
\hline
\end{tabular}




\section{Extention to Composite Materials}

In this section, the solution domain is represented by a bi-material $\Omega=\Omega_{1} \cup \bar{\Omega}_{2}$, where $\Omega_{1}=\left\{(x, y) \in \mathbb{R}^{2} \mid(0.5)^{2}<x^{2}+y^{2}<1\right\}$ and $\Omega_{2}=\left\{(x, y) \in \mathbb{R}^{2} \mid x^{2}+y^{2}<(0.5)^{2}\right\}$. So, the mathematical formulation of this problem is governed by two Laplace's equations, one in each of the two-dimenisonal bounded domains $\Omega_{1}$ and $\Omega_{2}$. The first equation is

$$
\nabla^{2} u_{1}=0, \quad \text { in } \Omega_{1}
$$

subject to the same boundary conditions (2) and (5) which make the problem the so-called 'complete-electrode model' (CEM), but equation (6) is replaced by

$$
\int_{\partial \Omega_{\text {Outer }}} u d s=0
$$

where $\partial \Omega_{\text {Outer }}=\left\{(x, y) \in \mathbb{R}^{2} \mid x^{2}+y^{2}=1\right\}$. The second Laplace's equation is

$$
\nabla^{2} u_{2}=0, \quad \text { in } \Omega_{2}
$$

subject to the following transmission conditions on the interface $\bar{\Omega}_{1} \cap \bar{\Omega}_{2}=\partial \Omega_{2}$ :

$$
u_{1}=u_{2}
$$

and

$$
\frac{\partial u_{1}}{\partial n_{1}}=-\gamma \frac{\partial u_{2}}{\partial n_{2}}
$$

where $\underline{n}_{1}$ is the outward unit normal to the boundary $\partial \Omega_{1}$ of the material $\Omega_{1}$ and $\underline{n}_{2}=-\underline{n}_{1}$ is the outward unit normal to the boundary $\partial \Omega_{2}$ of the material $\Omega_{2}$, and $0<\gamma \neq 1<\infty$ is the ratio between the two conductivities of the materials $\Omega_{2}$ and $\Omega_{1}$. In the formulation above, $\Omega_{2}$ is defined as a general inclusion and the geometry of the whole inclusion EIT problem is shown in Figure 15. The previous cases (simply-connected and multiply-connected) of Sections 5 and 6 could be considered as special cases of this composite material case, since:

(i) if $\gamma=1$, then the two composite material case becomes the simply-connected domain of Section 5 .

(ii) if $\gamma=\infty$, then the two composite material case becomes the annular domain with a rigid inclusion of Section 6.1.

(iii) if $\gamma=0$, then the two composite material case becomes the annular domain with a cavity of Section 6.2. 
Figure 15: The two-dimensional CEM in a composite domain, for $L=2$ and 4 electrodes.
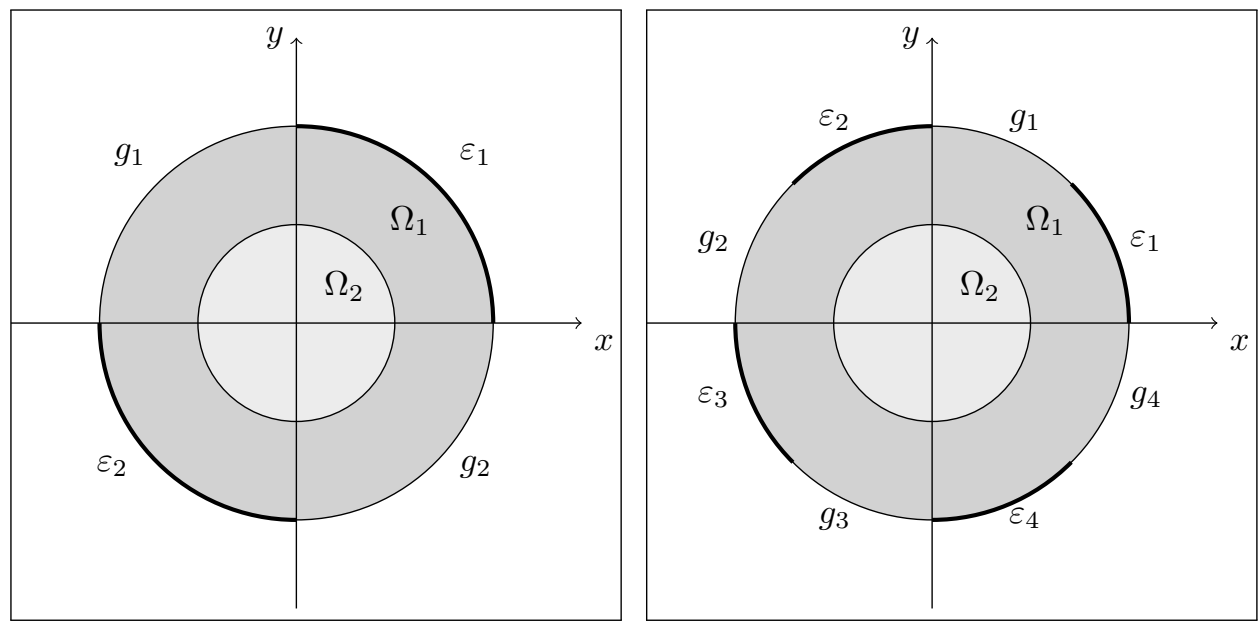

\subsection{Applying the BEM to the Direct EIT Problem in a Composite Bi-material}

In this section, we will use the BEM to solve the inclusion EIT problem given by equations (2), (5), (48)-(52). For the first domain $\Omega_{1}$, the discretisation of the boundary $\partial \Omega_{1}$, is the same as in Section 6.1. Hence, the BEM reduces the Laplace's equation (48) for $u_{1}$ to a new linear system of equations similar to (26), namely,

$$
B \underline{u_{1}}+A \underline{u_{1}^{\prime}}=\underline{0},
$$

where $\underline{u}_{1}:=\left(\begin{array}{l}{\frac{u_{1}}{\text { Outer }}}_{\underline{u}_{1} \text { Inner }}\end{array}\right):=\left(\begin{array}{c}u_{1}\left(\underline{\tilde{p}}_{i}\right)_{i=\overline{1, M}} \\ u_{1}\left(\underline{\tilde{p}}_{i}\right)_{i=\overline{M+1,2 M}}\end{array}\right)$

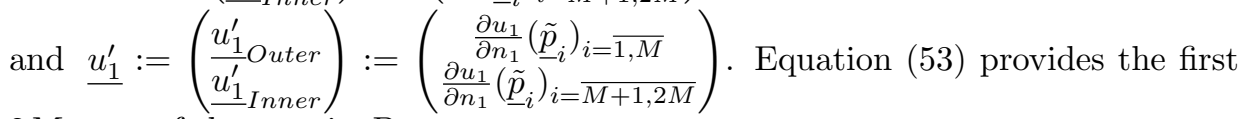
$2 M$ rows of the matrix $D$.

Now, for the second domain $\Omega_{2}$ we discretise the internal boundary $\partial \Omega_{2}$ into $M$ boundary elements, directed anticlockwise. Hence, the BEM reduces the second Laplace's equation (50) for $u_{2}$ to a new linear system of equations, similar to (8), namely,

$$
\tilde{B} \underline{u_{2}}+\tilde{A} \underline{u_{2}^{\prime}}=\underline{0},
$$

where $\underline{u_{2}}=\left(u_{2}\left(\tilde{p}_{2 M+1-i}\right)\right)_{i=\overline{M+1,2 M}}$ and $\underline{u_{2}^{\prime}}=\left(\frac{\partial u_{2}}{\partial n_{2}}\left(\tilde{p}_{2 M+1-i}\right)\right)_{i=\overline{M+1,2 M}}$. Collocating the interface transmission conditions (51) and (52) at the corresponding 
boundary element nodes and using (54) we obtain

$$
\tilde{B}\left(\underline{u_{1}}\right)_{\text {Inner }}-\frac{1}{\gamma} \tilde{A}\left(\underline{u_{1}^{\prime}}\right)_{\text {Inner }}=\underline{0} .
$$

Equations (53) and (55) from a system of $3 M$ equations with $4 M$ unkowns. In order to make this system of equations uniquely solvable the conditions (2), (5) and (49) should be imposed on the outer boundary. To begin with, we collocate the boundary condition (2) for the electrodes $\varepsilon_{p}, p=\overline{1, L}$, at the nodes $\tilde{p}_{(i-3 M)}$, resulting in

$$
\begin{aligned}
u_{1(i-3 M)}+z_{p} u_{1(i-3 M)}^{\prime} & -\frac{2 \pi}{M \ell_{p}} \sum_{k=(K M / L)+1}^{(2 K+1) M /(2 L)} u_{1 k}=\frac{z_{p} I_{p}}{\ell_{p}}, \\
i & =\overline{(3 M+1+K M / L),(3 M+(2 K+1) M /(2 L)},
\end{aligned}
$$

where $K=\overline{0,(L-1)}$. This yields $M / 2$ equations.

Second, by applying the zero flux boundary condition (5) on the gaps $g_{p}$, $p=\overline{1, L}$, between electrodes, we obtain

$$
u_{1(i-3 M)}^{\prime}=0, \quad i=\overline{(3 M+1+(2 K-1) M /(2 L)),(3 M+K M / L)},
$$

where $K=\overline{1, L}$. This yields another $M / 2$ equations.

Finally, the condition (6) yield one more equation, namely,

$$
\sum_{k=1}^{M} u_{1 k}=0
$$

Therefore, to find the solution of the CEM given by equations (2), (5) and (48)(52) in a composite material using the BEM, the equations (53), (55) and (56)-(58) are reformulated in the following matrix form as a $(4 M+1) \times(4 M)$ linear system of algebraic equations:

$$
D \underline{X}=\underline{b}
$$

where

$$
\underline{X}=\left(\begin{array}{l}
\underline{u}_{\text {Outer }} \\
\underline{u}_{\text {Inner }} \\
{\underline{u^{\prime}}}_{\text {Outer }} \\
{\underline{u_{\text {Inner }}^{\prime}}}_{\text {Inn }}
\end{array}\right) .
$$

Since the system of equations (59) is over-determined, we have used the leastsquares method to solve it. This yields the solution (17) for the unspecified boundary data (60). 


\subsection{Applying the MFS to the Direct EIT Problem in a Com- posite Bi-material}

In this section, the MFS for the Laplace's equations (48) and (50) in the composite material $\Omega=\Omega_{1} \cup \bar{\Omega}_{2}$ is applied by seeking a solution of Laplace's equation (48) as a linear combination of fundamental solutions of the form:

$$
u_{1}(\underline{p})=\sum_{j=1}^{2 N} c_{j} G\left(\underline{\xi}_{j}, \underline{p}\right), \quad \underline{p} \in \bar{\Omega}_{1}
$$

where the sources $\underline{\xi}_{j}$ and the collocation points $\underline{x}_{i}$ are exactly the same as in Section 6.2 , and by seeking a solution of Laplace's equation (50) as a linear combination of fundamental solutions of the form:

$$
u_{2}(\underline{p})=\sum_{j=2 N+1}^{3 N} c_{j} G\left(\underline{\xi}_{j}, \underline{p}\right), \quad \underline{p} \in \bar{\Omega}_{2}
$$

Similar domain decompositions for composite materials have been developed in [3-5] for the steady-state heat conduction governed by Laplace's equation, for the steady-state heat transfer in a fin governed by the modified Helmholtz equation, and for the steady-state elasticity governed by the Lame'system, respectively.

In (62), the sources $\underline{\xi}_{j}$ are located outside $\Omega_{\text {Inner }}$, so

$\underline{\xi}_{j}=\left(\xi_{j}^{1}, \xi_{j}^{2}\right)=\left(R_{2} \cos \left(\frac{2 \pi(j-2 N)}{N}\right), R_{2} \sin \left(\frac{2 \pi(j-2 N)}{N}\right)\right), j=\overline{2 N+1,3 N}$,

where $0.5<R_{2}<\infty$, and the new internal boundary collocation points are

$$
\underline{x}_{i}=\left(0.5 \cos \left(\frac{2 \pi(i-2 M)}{M}\right), 0.5 \sin \left(\frac{2 \pi(i-2 M)}{M}\right)\right), i=\overline{2 M+1,3 M} .
$$

In order to obtain the coefficient vector $\underline{c}=\left(c_{j}\right)_{j=\overline{1,3 N}}$, we substitute equations (9), (34), and (35) into the boundary conditions. To begin with, applying the boundary condition (2) results in equation (36), which in turn yields $M / 2$ equations. In addition, applying the zero flux boundary condition (5) we obtain (38). This yields an additional $M / 2$ equations.

Using the transmission conditions (51) and (52) results in

$$
\sum_{j=1}^{2 N} c_{j} G\left(\xi_{j}, \underline{x}_{i}\right)-\sum_{j=2 N+1}^{3 N} c_{j} G\left(\xi_{j}, \underline{x}_{i}\right)=0, \quad i=\overline{M+1,2 M}
$$

and

$$
\sum_{j=1}^{2 N} c_{j} G^{\prime}\left(\xi_{j}, \underline{x}_{i}\right)-\hat{K} \sum_{j=2 N+1}^{3 N} c_{j} G^{\prime}\left(\xi_{j}, \underline{x}_{i}\right)=0, \quad i=\overline{2 M+1,3 M},
$$


respectively. These give $2 M$ equations.

Finally, by imposing the condition (49), yields one more equation

$$
\sum_{i=1}^{2 M} \sum_{j=1}^{2 N} c_{j} G\left(\underline{\xi}_{j}, \underline{x}_{i}\right)=0
$$

Again, to find the solution of the CEM problem (2), (5) and (48)-(52) using the MFS, the equations (61)-(65) are reformulated in the following matrix form as a $(3 M+1) \times 3 N$ linear system of algebraic equations:

$$
F \underline{c}=\underline{b} .
$$

The least-squares method is used to solve the system of equations (66). This yields the solution (24).

Example 5. Solve the problem (2), (5) and (48)-(52) using the BEM and MFS with the same input data as in Example 1 and $\gamma=2$.

BEM solution: The matrix $D$ in equation (59) is given by

$$
D_{i, l}= \begin{cases}B_{i, l} & \text { if } l=\overline{1,2 M}, \\ A_{i, l} & \text { if } l=\overline{(2 M+1), 4 M}, \quad i=\overline{1,2 M},\end{cases}
$$

and

$$
D_{i, l}=\left\{\begin{array}{ll}
\tilde{B}_{i, l} & \text { if } l=\overline{M+1,2 M}, \\
\tilde{A}_{i, l} & \text { if } l=\overline{(3 M+1), 4 M}, \\
0 & \text { if } l=\overline{1, M(2 M+1), 3 M},
\end{array} \quad i=\overline{(2 M+1), 3 M} .\right.
$$

On the other hand, using equations (56)-(58) we obtain

$$
\begin{aligned}
& D_{i, l}= \begin{cases}-\frac{h}{\ell_{1}} & \text { if }(i-3 M) \neq l, l=\overline{1, M / 4} \\
\left(1-\frac{h}{\ell_{1}}\right) & \text { if }(i-3 M)=l, l=\overline{1, M / 4} \\
0 & \text { if } l=\overline{(M / 4+1), 2 M} \cup \overline{(3 M+1), 4 M} \\
z_{1} \delta_{i, l} & \text { if } l=\overline{(2 M+1), 3 M},\end{cases} \\
& i=\overline{(3 M+1),(3 M+M / 4)}, \\
& D_{i, l}= \begin{cases}-\frac{h}{\ell_{2}} & \text { if }(i-3 M) \neq l, l=\overline{(M / 2+1), 3 M / 4} \\
\left(1-\frac{h}{\ell_{2}}\right) & \text { if }(i-3 M)=l, l=\overline{(M / 2+1), 3 M / 4} \\
0 & \text { if } l=\overline{1, M / 2} \cup \overline{(3 M / 4+1), M} \cup \overline{(3 M+1), 4 M} \\
z_{2} \delta_{i, l} & \text { if } l=\overline{(2 M+1), 3 M},\end{cases} \\
& i=\overline{(3 M+M / 2+1),(3 M+3 M / 4)}, \\
& D_{i, l}=\delta_{i, l}, \quad \text { if } l=\overline{(2 M+1), 3 M} \text {, }
\end{aligned}
$$




$$
i=\overline{(3 M+M / 4+1),(M+M / 2)} \cup \overline{(2 M+3 M / 4+1), 4 M} .
$$

The last row in the matrix $D$ is given by

$$
D_{(4 M+1), l}= \begin{cases}1 & \text { if } l=\overline{1, M} \\ 0 & \text { if } l=\overline{M+1,4 M} .\end{cases}
$$

Furthermore, the vector $\underline{b}$ is given by (41).

MFS solution: Turning now to the MFS solution, the first $M$ rows of the matrix $F$ in equation (66) are the same as those of the matrix $F$ in Example 1. Moreover, another $M$ rows are generated by applying the inner boundary condition (64), namely,

$$
\begin{gathered}
F_{i, j}=G_{i, j}, \quad i=\overline{(M+1), 2 M}, \quad j=\overline{1,2 N} \\
F_{i, j}=-G_{i, j-N}, \quad i=\overline{(M+1), 2 M}, \quad j=\overline{2 N+1,3 N} .
\end{gathered}
$$

Another $M$ rows in the matrix $F$ are obtained from (64) as

$$
\begin{gathered}
F_{i, j}=G_{i, j}^{\prime}, \quad i=\overline{(2 M+1), 3 M}, \quad j=\overline{1,2 N}, \\
F_{i, j}=2 G_{i, j-N}^{\prime}, \quad i=\overline{(2 M+1), 3 M}, \quad j=\overline{2 N+1,3 N} .
\end{gathered}
$$

Finally, the last row in the matrix $F$ is obtained from (65) as

$$
\begin{aligned}
& F_{(3 M+1), j}=\sum_{i=1}^{2 M} G_{i, j}, \quad j=\overline{1, N} \\
& F_{(3 M+1), j}=0, \quad j=\overline{N+1,3 N} .
\end{aligned}
$$

Similarly, the vector $\underline{b}$ of the linear system of equations (66) is given by (43). In the MFS we take $R=1.15, R_{1}=0.45$ and $R_{2}=0.55$.

Figures 16-19 present a comparison between the BEM and MFS solutions for the boundary data $u_{\text {Outer }}(1, \theta), u_{\text {Inner }}(0.5, \theta),(\partial u / \partial n)_{\text {Outer }}(1, \theta)$ and $(\partial u / \partial n)_{\text {Inner }}(0.5, \theta)$, respectively. The same conclusions as in Example 3 can be drawn by observing these figures. 

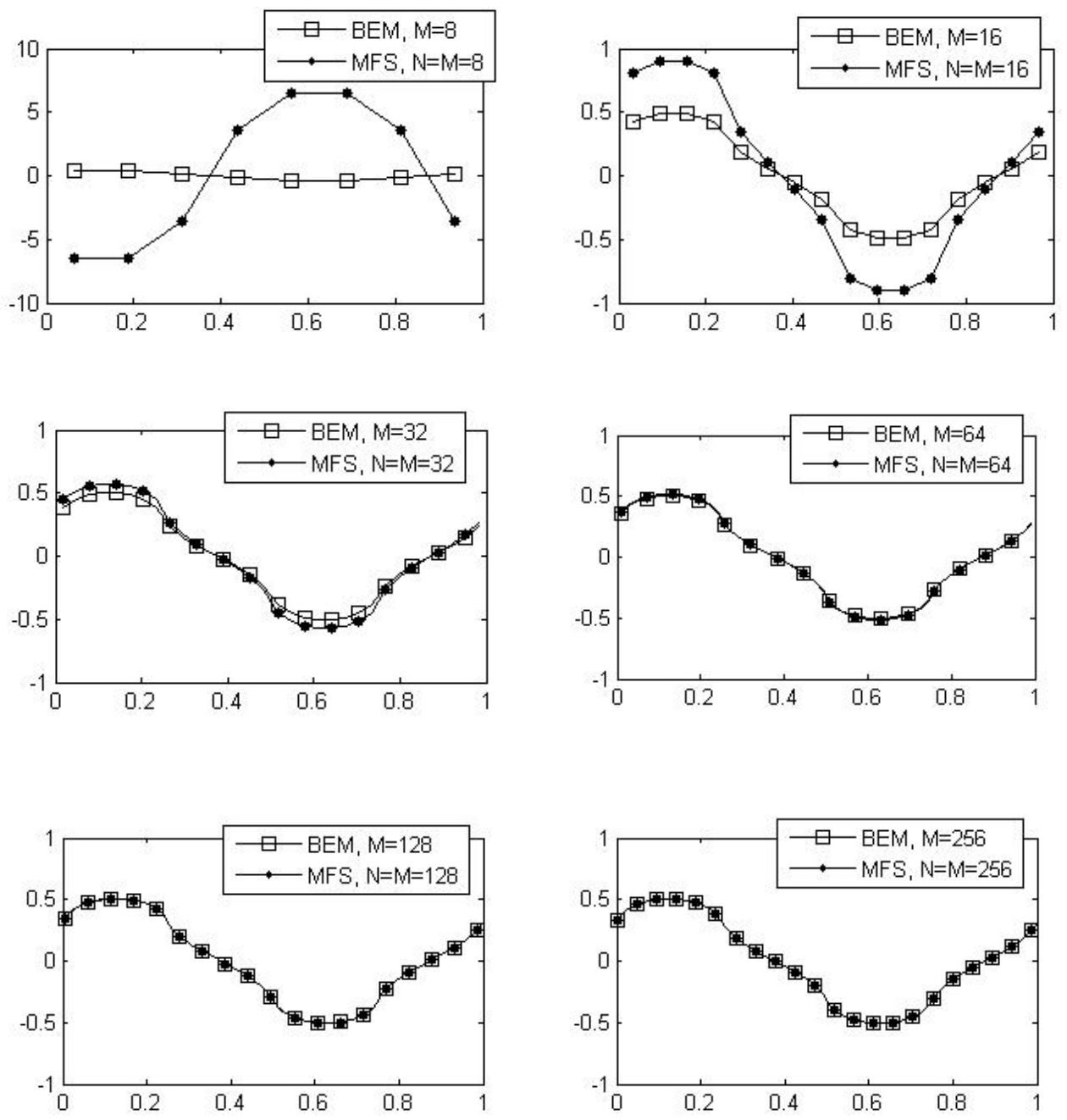

Figure 16: Comparison between $u_{\text {Outer }}^{M F S}(1, \theta)$ and $u_{\text {Outer }}^{B E M}(1, \theta)$, as functions of $\theta /(2 \pi)$, for Example 5 . 

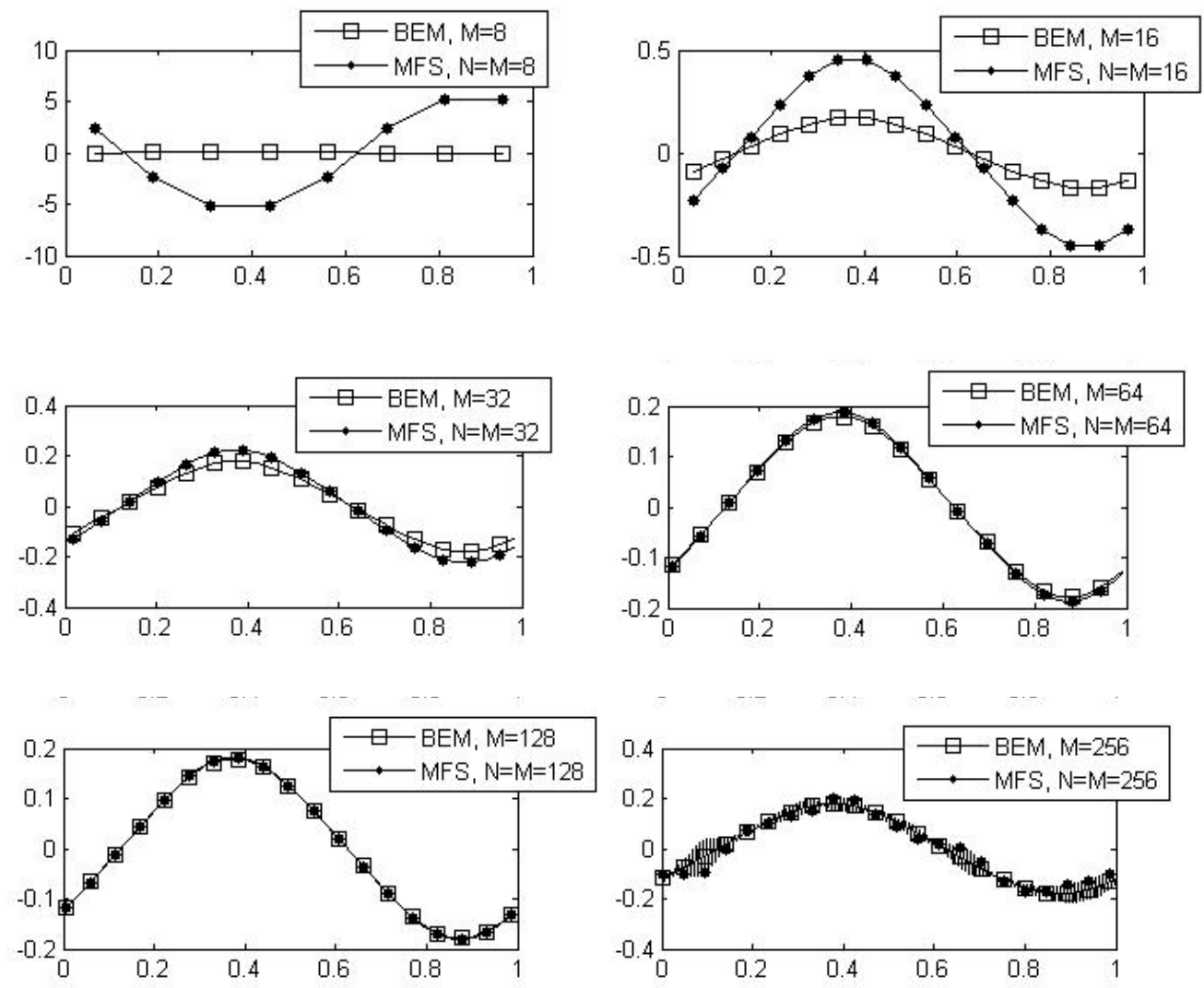

Figure 17: Comparison between $u_{\text {Inner }}^{M F S}(0.5, \theta)$ and $u_{\text {Inner }}^{B E M}(0.5, \theta)$, as functions of $\theta /(2 \pi)$, for Example 5. 

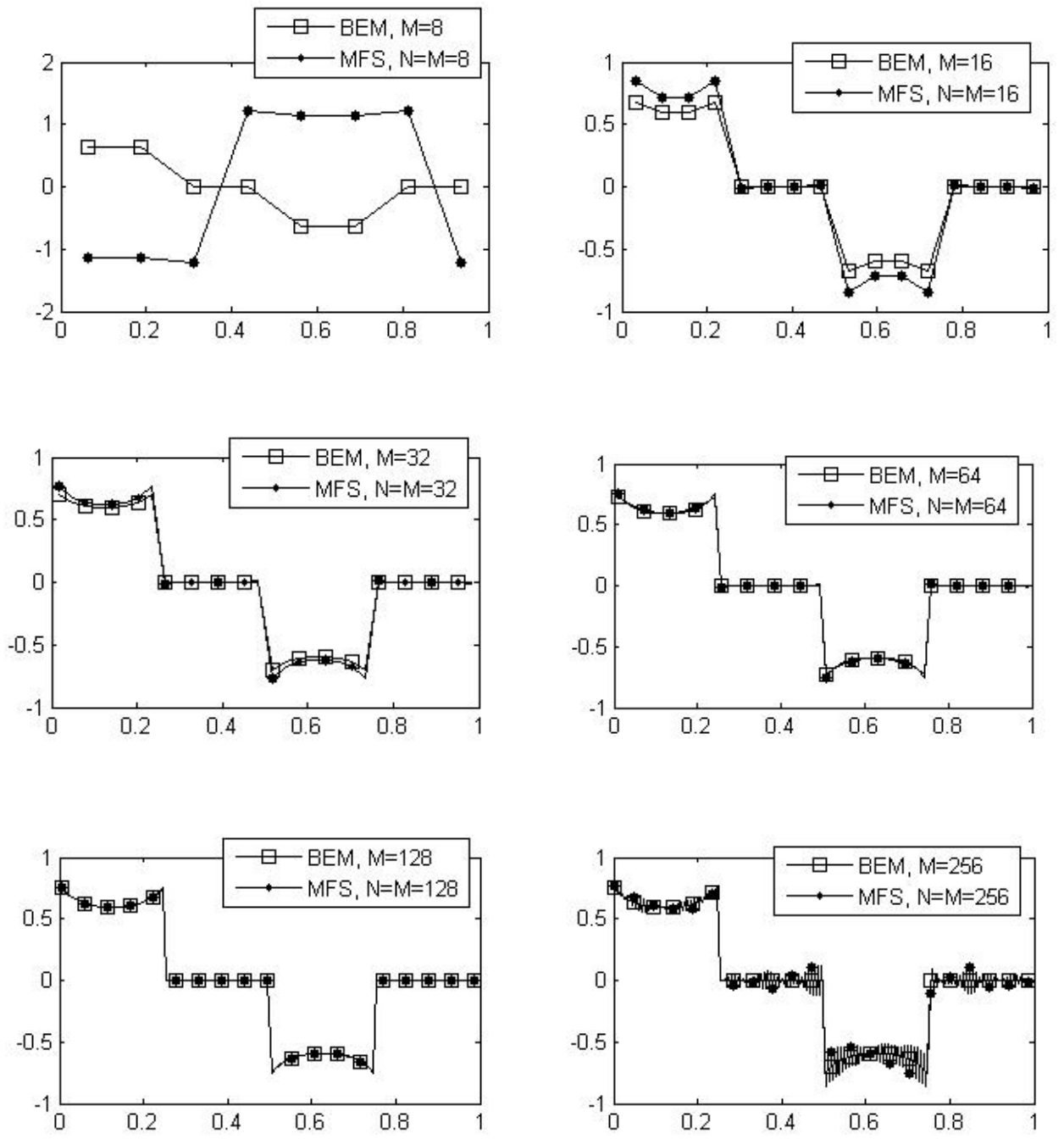

Figure 18: Comparison between $\left(\frac{\partial u}{\partial n}\right)_{\text {Outer }}^{M F S}(1, \theta)$ and $\left(\frac{\partial u}{\partial n}\right)_{\text {Outer }}^{B E M}(1, \theta)$, as functions of $\theta /(2 \pi)$, for Example 5 . 

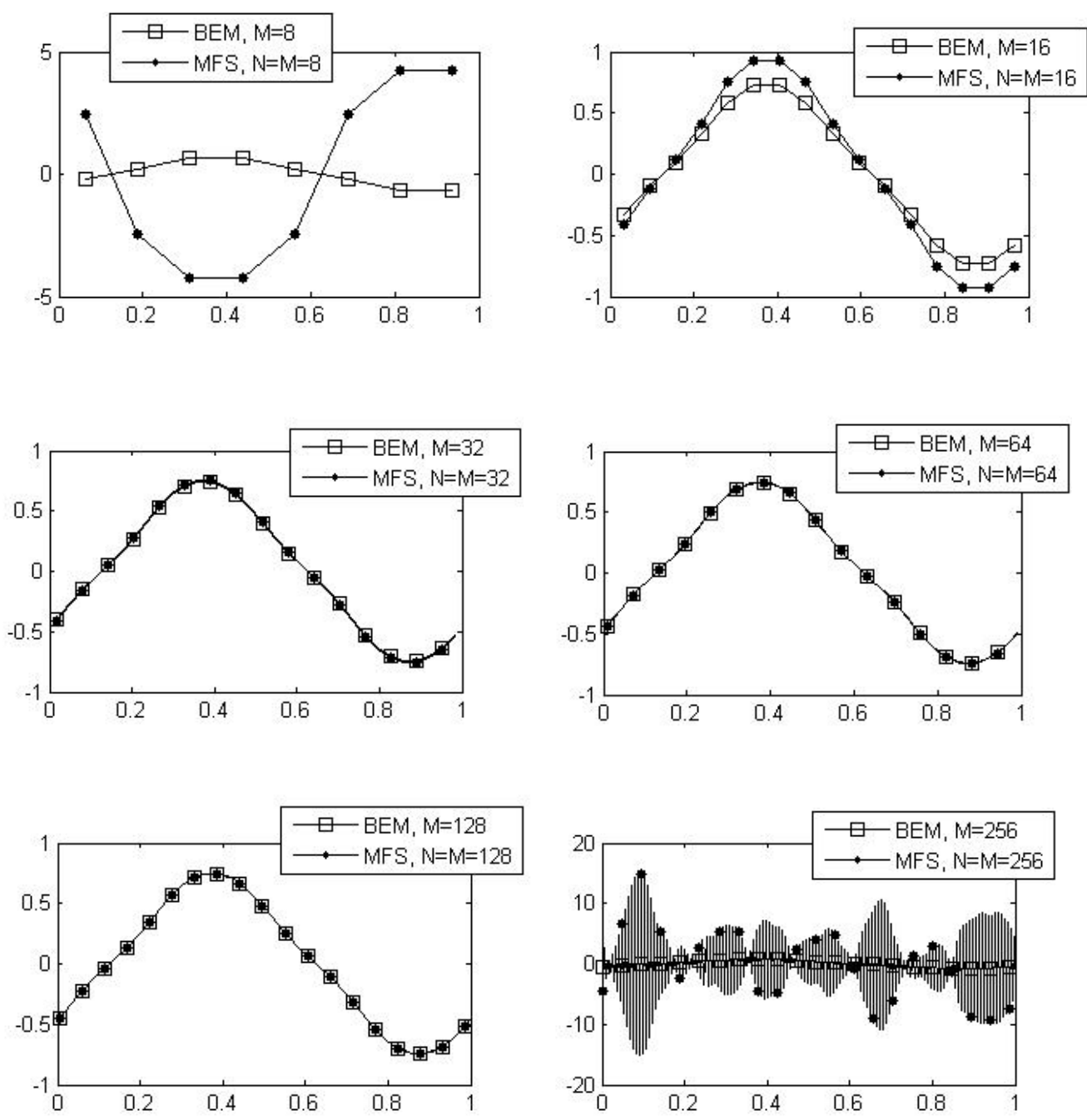

Figure 19: Comparison between $\left(\frac{\partial u}{\partial n}\right)_{\text {Inner }}^{M F S}(0.5, \theta)$ and $\left(\frac{\partial u}{\partial n}\right)_{\text {Inner }}^{B E M}(0.5, \theta)$, as functions of $\theta /(2 \pi)$, for Example 5 .

\section{Conclusions}

This study has applied and compared the BEM and MFS to solve the completeelectrode model (CEM) problem of ERT. These two numerical methods were examined for various simply and multiply-connected domains with various homogeneous boundary conditions on the inner boundary in the latter case. Due to 
the lack of an analytical solution, the BEM solution has been considered as the exact solution because it is more accurate than the one obtained using the MFS which gives some instability when the degrees of freedom become too large. The boundary integrals involved in the BEM have been evaluated analytically. As far as the computational time is concerned, both the BEM and the MFS require almost the same modest amount of time (mainly used to invert the linear systems of equations (16) or (23)); e.g. 3,5 and 30 seconds for $M \in\{64,128,256\}$ boundary elements, respectively. Another interesting point to make is that in the MFS we have experienced with various values of $R>1$ and we have found that $R$ between 1.01 and 1.15 produces the most accurate results. For larger values of $R$, the MFS accuracy decreases showing that the harmonic function $u$ outside the unit disk domain $\Omega$ has reached its limit, i.e. the circle of radius $R$ captured in its interior a singularity of $u$. The nature of the Robin boundary condition (2) and, in general, the sophisticated CEM makes it difficult to predict analytically beforehand where the singularities of $u$ lie in the exterior of $\Omega$. In any case, $R$ should be chosen less that the magnitude of position vector of the nearest singularity to the origin. Although the MFS has produced unstable solutions for large degrees of freedom, such as $M=N=256$, for lower values its accuracy and stability are excellent when compared to the BEM numerical solution. Moreover, the MFS is much easier to implement than the BEM especially in three-dimensional problems in irregular domains. Immediate future work consists in applying the direct methods developed in this study to the inverse problem of the ERT/EIT.

\section{Acknowledgement}

T. E. Dyhoum would like to thank the Libyan Ministry of Higher Education and Libyan Embassy for their financial support in this research.

\section{References}

[1] Aykroyd, R. G. and Cattle, B. A. A boundary-element approach for the complete-electrode model of EIT illustrated using simulated and real data, Inverse Problems in Science and Engineering, 15, 441-461, 2007.

[2] Aykroyd, R. G., Cattle, B. A. and West, R. M. Boundary element method and Markov chain Monte Carlo for object location in electrical impedence tomography, 5th International Conference on Inverse Problems in Engineering: Theory and Practice, (ed. D. Lesnic), Chapter A09, Vol. II, (5 pages), 2005.

[3] Berger, J. R. and Karageorghis, A. The method of fundamental solutions for heat conduction in layered materials, International Journal for Numerical Methods in Engineering, 45, 1681-1694,1999.

[4] Berger, J. R. and Karageorghis, A. The method of fundamental solutions for heat conduction in layered elastic materials, Engineering Analysis with Boundary Elements, 25, 877-886, 2001. 
[5] Bin-Mohsin, B. and Lesnic, D. The method of fundamental solutions for Helmholtz-type equations in composite materials, Computers and Mathematics with Applications, 62, 4377-4390, 2011.

[6] Bogomolny, A. Fundamental solution method for elliptic boundary value problems, SIAM Journal on Applied Mathematics, 22, 644-669, 1985.

[7] Chen, C., Cho, H. and Golberg, M. Some comments on the ill-conditioning of the method of fundamental solutions, Engineering Analysis with Boundary Elements, 30, 405-410, 2006.

[8] Cheng, K. S., Isaacsson, D., Newell, J. C. and Gisser, D. G. Electrode models for electric current computed tomography, IEEE Transactions on Biomedical Engineering, 36, 918-924, 1989.

[9] Delbary, F. and Kress, R. Electrical impedance tomography using a point electrode inverse scheme for complete electrode data, Inverse Problems and Imaging, 5, 355-369, 2011.

[10] Dong, G., Zou, J., Bayford, R.H., Ma, X., Gao, S., Yan, W. and Ge, M. The comparison between FVM and FEM for EIT forward problem, IEEE Transactions on Magnetics, 41, 1468-1471, 2005.

[11] Heravi, M. A., Marin, L. and Sebu, C. The method of fundemental solutions for complex electrical impedance tomography, Engineering Analysis with Boundary Elements, 2014, (submitted).

[12] Hu, G., Chen, M., He, W. and Zhai, J. A novel forward problem solver based on meshfree method for electrical impedance tomography, Przeglad Elektrotechniczny, 89, No.2a, 234-237, 2013.

[13] Katsikadelis, J. Boundary Elements: Theory and Applications, 1st ed., New York: Elsevier, 2002.

[14] Karageorghis, A. and Lesnic, D. Detection of cavities using the method of fundamental solutions, Inverse Problems in Science and Engineering, 17, 803820, 2009.

[15] Karageorghis, A. and Lesnic, D. The fundamental solutions for inverse conductivity problem, Inverse Problems in Science and Engineering, 18, 567-583, 2010 .

[16] Kim, S., Wang, R. L., Khambampati, A. K., Lee, B. A and Kim, K. Y. An improved boundary distributed source method for electrical resistance tomography forward problem, Engineering Analysis with Boundary Elements, 2014, (in press).

[17] Kitagawa, T. On numerical stability of the method of fundamental solutions applied to the Dirichlet problem, Japan Journal of Applied Mathematics, 5, 123-133, 1988. 
[18] Knudsen, K. On the Inverse Conductivity Problem, Ph.D. Thesis, Department of Mathematical Sciences, Aalborg University, Denmark, 2002.

[19] Lesnic, D., Elliott, L. and Ingham, D. B. The boundary element solution of the Laplace and biharmonic equations subjected to noisy boundary data, International Journal for Numerical Methods in Engineering, 43, 479-492, 1998 .

[20] Pidcock, M. K., Kuzuoglu, M. and Leblebicioglu, K. Analytic and semianalytic solutions in electrical impedance tomography: I. Two-dimensional problems, Physiological Measurements, 16, 77-90, 1995.

[21] Ramachandran, P. A. Method of fundamental solutions: singular value decomposition analysis, Communications in Numerical Methods in Engineering, 18, 789-801, 2002.

[22] Rolnik, V., Menin, O. H., Carosio, G. L. C. and Junior, P. S. Study of the boundary element method for the direct problem of electrical impedance tomography, ABCM Symposium Series in Mechatronics, 4, 835-843, 2010.

[23] Soleimani, E., Powell, C. E. and Polydorides, N. Improving the forward solver for the complete electrode model in EIT using algebraic multigrid, IEEE Transactions on Medical Imaging, 24, 577-583, 2005.

[24] Somersalo, E., Cheney, M. and Isaacson, D. Existence and uniqueness for electrode models for electric current computed tomography, SIAM Journal on Applied Mathematics, 52, 1023-1040, 1992.

[25] Tarvainen, M., Vauhkonen, M., Savolainen, T. and Kaipio, J. P. Boundary Element Method and Internal Electrodes in Electrical Impedance Tomography, Department of Applied Physics, University of Kuopio, Report No. 17, 2000.

[26] Vilhunen, T., Kaipio, J. P., Vauhlonen, P. P., Savolainen, T. and Vauhkonen, M. Simultaneous reconstruction of electrode contact impedance and internal electrical properties: I. Theory, Measurement Science and Technology, 13, 1848-1854, 2000. 